\title{
Uncertainty analysis of projections of ozone-depleting substances: mixing ratios, EESC, ODPs, and GWPs
}

\author{
G. J. M. Velders ${ }^{1}$ and J. S. Daniel ${ }^{2}$ \\ ${ }^{1}$ National Institute for Public Health and the Environment (RIVM), PO Box 1, 3720 BA Bilthoven, the Netherlands \\ ${ }^{2}$ National Oceanic and Atmospheric Administration, Earth System Research Laboratory, Chemical Sciences Division, \\ Boulder, CO 80305, USA
}

Correspondence to: G. J. M. Velders (guus.velders@rivm.nl)

Received: 12 September 2013 - Published in Atmos. Chem. Phys. Discuss.: 29 October 2013

Revised: 22 January 2014 - Accepted: 8 February 2014 - Published: 17 March 2014

\begin{abstract}
The rates at which ozone-depleting substances (ODSs) are removed from the atmosphere, which determine the lifetimes of these ODSs, are key factors for determining the rate of ozone layer recovery in the coming decades. We present here a comprehensive uncertainty analysis of future mixing ratios of ODSs, levels of equivalent effective stratospheric chlorine (EESC), ozone depletion potentials, and global warming potentials (GWPs), using, among other information, the 2013 WCRP/SPARC (World Climate Research Programme/Stratospheric Processes and their Role in Climate) assessment of lifetimes of ODSs and their uncertainties. The year EESC returns to pre-1980 levels, a metric commonly used to indicate a level of recovery from ODSinduced ozone depletion, is 2048 for midlatitudes and 2075 for Antarctic conditions based on the lifetimes from the SPARC assessment, which is about 2 and $4 \mathrm{yr}$, respectively, later than based on the lifetimes from the WMO (World Meteorological Organization) assessment of 2011. However, the uncertainty in this return to 1980 levels is much larger than the shift due to this change in lifetimes. The year EESC returns to pre-1980 levels ranges from 2039 to 2064 (95\% confidence interval) for midlatitudes and from 2061 to 2105 for the Antarctic spring. The primary contribution to these ranges comes from the uncertainty in the lifetimes, with smaller contributions from uncertainties in other modeled parameters. The earlier years of the return estimates derived by the uncertainty analysis, i.e., 2039 for midlatitudes and 2061 for Antarctic spring, are comparable to a hypothetical scenario in which emissions of ODSs cease in 2014. The later end of the range, i.e., 2064 for midlatitudes and 2105 for Antarctic spring, can also be obtained by a scenario with an
\end{abstract}

additional emission of about $7 \mathrm{Mt}$ CFC-11 eq. (eq. - equivalent) in 2015, which is the same as about 2 times the projected cumulative anthropogenic emissions of all ODSs from 2014 to 2050 , or about 12 times the projected cumulative HCFC emissions from 2014 to 2050.

\section{Introduction}

Projections of the mixing ratios of ozone-depleting substances (ODSs) and the levels of chlorine and bromine in the stratosphere require knowledge of future production and loss processes in addition to current atmospheric abundances and amounts of ODSs present in existing equipment. In the past five WMO/UNEP (World Meteorological Organization/United Nations Environment Programme) Scientific Assessments of Ozone Depletion (WMO, 1995, 1999, 2003, 2007, 2011) a box model has been used to calculate mixing ratios and equivalent effective stratospheric chlorine (EESC) from historic data and future projections of ODSs. EESC is an index that relates the time evolution of long-lived ODSs in the troposphere with their ability to destroy stratospheric ozone (Daniel et al., 1995, 2010; WMO, 2011). Uncertainties in parameters and their effects on mixing ratios and EESC levels have not received much attention. Newman et al. (2007) reformulated EESC by using the age-of-air spectrum and age-of-air dependent fractional release values and analyzed the effects of uncertainty in these parameters on EESC. They found that the largest uncertainties in EESC are associated with the mean age of air in the stratosphere and the fractional release of halogens from ODSs in the stratosphere. 
Daniel et al. (2007) studied the effects of different assumptions for the amounts of ODSs present in existing equipment on future EESC levels. One of the key pieces of information for calculating future mixing ratios and EESC and for estimate past ODS emissions from observed mixing ratios is knowledge of the ODS lifetimes; yet, to our knowledge, an uncertainty analysis has not been performed with respect to lifetime uncertainties. Douglass et al. (2008) suggested that past estimates of the CFC (chlorofluorocarbon)-11 lifetime were too short and that longer lifetimes are found by models that more accurately simulate atmospheric circulation and the age of air in the stratosphere. The WMO/UNEP assessment (WMO, 2011) indeed concluded that there was emerging evidence that the lifetimes of some important ODSs (e.g., CFC-11) may be somewhat longer than previously reported (Montzka and Reimann, 2011). Since then several papers have been published on lifetimes of ODSs (Laube et al., 2013; Minschwaner et al., 2013; Rigby et al., 2013; Brown et al., 2013) and an assessment of lifetimes has been conducted by the World Climate Research Programme (WCRP) Stratospheric Processes And their Role in Climate (SPARC) project (SPARC, 2013). In the SPARC assessment, a new set of recommended lifetimes were presented based on calculations with atmospheric chemistry-transport models, analysis of observations at the surface and in the stratosphere, laboratory analysis of chemical reactions and photolysis rates, and on inverse modeling. Apart from a new set of lifetimes, a complete uncertainty analysis of lifetimes for each of the major halogenated ODSs was presented for the first time. The uncertainties in the lifetimes are considerable, ranging from 3 to $33 \%(1 \sigma)$, with significant implications for projecting the decay of ODS concentrations in the atmosphere. The new lifetimes and associated uncertainties and their effects on future EESC levels are the basis of the analysis presented here.

Four items are explored. First, the effects of the new SPARC (2013) lifetimes on future mixing ratios of ODSs and EESC levels are evaluated by comparing these values with those calculated using the lifetimes from WMO (2011). Second, the effects of the uncertainties in the lifetimes on mixing ratios and EESC levels are discussed. Third, a complete uncertainty analysis is performed for the calculations of EESC levels and the year of return to 1980 EESC levels as well as for estimates of ODS radiative forcing, by considering uncertainties and correlations in uncertainties of all the relevant parameters. Fourth, ozone-depletion potentials (ODPs), global warming potentials (GWPs), and their uncertainties are calculated using the new lifetime information.

Here we focus on the long-lived chlorine and bromine containing species and EESC as a measure of the amount of active chlorine/bromine in the stratosphere available to cause ozone depletion. We recognize that other factors are also important for the future development of the ozone layer, such as future concentrations of nitrous oxide $\left(\mathrm{N}_{2} \mathrm{O}\right)$, methane $\left(\mathrm{CH}_{4}\right)$, very short-lived halogen containing species, and changes in temperature and circulation in the atmosphere. These other factors are not considered here.

The model, input data, lifetimes, and uncertainties in input parameters are discussed in Sect. 2. The results of the box model calculations and the effects of the uncertainties in the lifetimes and other parameters on mixing ratios and EESC are presented in Sect. 3. Uncertainties in the radiative forcing of ODSs are found in Sect. 4. In Sect. 5 and 6 uncertainties in ODPs and GWPs are presented. A discussion of the results follows in Sect. 7 and the conclusions in Sect. 8.

\section{Model and input description}

\subsection{Box model}

For the calculation of mixing ratios and EESC the box model and data are almost identical to what was used in the Scientific assessment of ozone depletion: 2010 (Daniel and Velders, 2011). There are only a few minor exceptions. One difference is the use of the age-of-air spectrum from Newman et al. (2007) and age-of-air dependent, fractional release values from Newman et al. (2006) in the calculation of EESC here. These fractional release values have also been used in WMO (2011), but only for discrete ages of air in midlatitude (3 yr) and Antarctic (5.5 yr) conditions. These changes in methodology are necessary for the Monte Carlo uncertainty analysis. It should be noted that the fractional release of any particular ODS varies throughout the stratosphere and that generally more of the source gas will have been destroyed in air that has been longer in the stratosphere (Hall, 2000). Fractional release is a simplistic way to relate the relative contribution of each source gas to the total equivalent chlorine at any stratospheric location or to the particular stratospheric age of air (i.e., the time the air parcel has resided in the stratosphere). The age of air appropriate for the midlatitude, lower stratosphere is about $3 \mathrm{yr}$. Thus, the fractional release values associated with 3 yr-old air are generally used for calculating midlatitude EESC that is appropriate for midlatitude stratospheric ozone depletion; values associated with $5.5 \mathrm{yr}$-old air are appropriate for representing springtime polar conditions (Newman et al., 2006, 2007). In this work, we use these two air ages to calculate EESC at midlatitudes and for springtime Antarctic conditions, but also incorporate variations from these fixed values in incorporating the age spectra of stratospheric air in the uncertainty analysis. While we refer to these two representative conditions, it should be remembered that fractional release values, and thus EESC, experience a range of values throughout the stratosphere and that it is incorrect to think of these as single fixed values over a range of locations for any given time. Furthermore, circulation and other variability lead to variability in both fractional release and EESC. It should also be recognized that in the semiempirical ODP formula (Sect. 5) the key quantity is the ratio of the fractional release of an ODS to that of CFC-11; 
this ratio tends to be a more robust quantity for a range of ages than do the fractional release values themselves.

In the box model, EESC is calculated for midlatitude and Antarctic conditions from surface mixing ratios. The surface global mean mixing ratio of specific ODS $i$ is calculated using Eq. (1),

$\frac{d \rho_{i}}{d t}=F_{i} E_{i}-\frac{\rho_{i}}{\tau_{i}}$

where $\rho_{i}$ is the mean surface mixing ratio (ppt - parts per trillion), $\tau_{i}$ is the total lifetime (years), $E_{i}$ is the emission rate $\left(\mathrm{kg} \mathrm{yr}^{-1}\right) . F_{i}\left(\mathrm{ppt} \mathrm{kg}^{-1}\right)$ is a factor that relates the mass emitted to the global mean mixing ratio as expressed by Eq. (2),

$F_{i}=\frac{N_{\mathrm{A}} F_{\text {surf }}}{M_{i} N_{\mathrm{atm}}}=\frac{F_{\text {surf }} \times 5.68 \times 10^{-9}}{M_{i}}$,

where $M_{i}$ is the molecular weight $\left(\mathrm{kg} \mathrm{mole}^{-1}\right), N_{\mathrm{A}}$ the Avogadro constant, $N_{\mathrm{atm}}$ the number of molecules in the global atmosphere, and $F_{\text {surf }}$ is a factor relating the global mean surface mixing ratio to the global mean atmospheric mixing ratio. The factor $F_{\text {surf }}$ is meant to account for the general decrease of the ODS mixing ratios with altitude above the tropopause, and for some molecules like $\mathrm{CH}_{3} \mathrm{Br}$, even in the troposphere. $F_{\text {surf }}$ was taken to be 1.07 for all ODSs (WMO, 2007, 2011), except for $\mathrm{CH}_{3} \mathrm{Br}$ for which 1.16 was taken as in WMO (2011) (see Table 2). Using a constant for $F_{\text {surf ne- }}$ glects changes in this factor that could be caused by changes in circulation or by the variability of the surface emission (and the resulting variability in the atmospheric vertical distribution).

For the emissions in the model a different approach is used for historic emissions than for future emissions. As in WMO (2011), historical annual emissions are derived, using Eq. (1), from observed mixing ratios and the lifetimes of the ODSs. This so-called top-down approach is taken because the ODSs are long-lived and the global mixing ratios and lifetimes are thought to be known more accurately than are independently derived bottom-up emissions. Future emissions are estimated from a scenario of future production, following the maximum allowed production under the Montreal Protocol (in combination with an extrapolation of observed trends over a few years for HCFCs), and the amounts of ODSs present in existing equipment and applications, generally called "banks". Banks here are defined as the quantity of ODSs produced but not yet emitted to the atmosphere. These quantities will be present mostly in refrigeration and air conditioning units and foams, but can also be in landfills. Future annual production is added directly to the bank in each respective year. Future emissions are then estimated directly from future bank sizes by assuming the fractional rate of release from the banks (emission factor) for each ODS remains the same as it has been when averaged over the previous $10 \mathrm{yr}$ (1999-2008). The present bank values could be determined from historical estimates of cumulative production and emission, with the differences between the cumulative values through today representing the current banks. However, because the bank sizes are generally small compared with these cumulative and somewhat uncertain values, the uncertainties in this approach could be large. Therefore, the bank sizes for a starting year (2008 here) are based on so-called bottom-up estimates from inventories of equipment containing a particular ODS (IPCC/TEAP, 2005; UNEP, 2009). With annual reported production and top-down derived emissions the banks can be calculated backwards and forwards in time starting from this 2008 point. For historical bank estimates in years further away from 2008, it is expected that bank uncertainties will increase because of the previously mentioned uncertainties in the cumulative production and emission numbers. However, by doing the analysis in this way, this uncertainty primarily affects our results through the emission factor as each year uncertainties in the bank size will affect the estimate of the emission factor.

The following step-by-step approach is used to obtain a mixing ratio time series from 1950 to 2120 . First, historical top-down emissions from 1979 to 2008 are derived from observed mixing ratios and lifetimes. Emissions and mixing ratios before 1979 were derived from a combination of bottom-up emission estimates and top-down estimates from mixing ratios extrapolated backward in time (WMO, 2003, 2007, 2011). Second, bank sizes are calculated backwards starting in 2008 using reported production data and top-down emissions. Third, emission factors for future emissions are calculated as the $10 \mathrm{yr}$ average of the fraction of the emission over the bank (1999-2008). Fourth, projections of mixing ratios are calculated using the box model (Eq. 1) out to 2120.

EESC is calculated using these mixing ratios, fractional release values, a time lag (age of air) for air to be transported from the surface to the relevant region of the stratosphere, and a factor $\alpha$ to account for the relative effectiveness of bromine compared with chlorine for ozone depletion. A value of 60 is used for $\alpha$ for midlatitude conditions and 65 for Antarctic springtime conditions (WMO, 2007, 2011). The single values for the $3 \mathrm{yr}$ age of air for midlatitude conditions and $5.5 \mathrm{yr}$ for Antarctic conditions, used in WMO (2011), are replaced by age-of-air spectra from Newman et al. (2007) with the same mean ages as before. The fractional release values of the ODSs corresponding to the 3 and $5.5 \mathrm{yr}$ mean ages of air are replaced by functions describing the age-of-air dependence of the fractional releases (Newman et al., 2006). These functions yield the same values for the 3 and $5.5 \mathrm{yr}$ ages of air as those used in WMO (2011), except for two. As argued in WMO (2011), the abundances of HCFC-141b and HCFC-142b were small and had large temporal trends at the time the measurements upon which these fractional release values are based were made, leading to large uncertainties. Therefore, the functions describing the age-of-air dependent fractional release values for HCFC$141 \mathrm{~b}$ and HCFC-142b were not used in WMO (2011), but 
instead values from WMO (2007) were used. Here, age-ofair dependent fractional release values for HCFC-141b and HCFC-142b are used by adjusting the functions of Newman et al. (2006) to be in better agreement with the values used in WMO $(2011,2007)$ for 3 and $5.5 \mathrm{yr}$ mean ages and with the curves of other ODS (see footnote in Table 2).

Recently new fractional release values with associated uncertainties have been reported by Laube et al. (2013). The absolute values derived by Laube et al. (2013) are about $20 \%$ smaller than those derived by Newman et al. (2006), and those used in WMO (2011). Although this $20 \%$ does result in smaller EESC levels, it hardly changes the EESC curve shape, and the year EESC returns to pre-1980 levels is only changed by about $1 \mathrm{yr}$ for midlatitude conditions. For Antarctic spring conditions the year EESC returns to pre-1980 levels is about 5 yr earlier with the fractional release values from Laube et al. (2013) than with those from WMO (2011). As discussed by Laube et al. (2013), the differences in fractional release values are probably caused by differences in the determination of the age of air in the measurements and by interannual variability in the fractional release of the halogens. Since both sets of fractional release values yield about the same EESC time series shape, we choose to use the recommended fractional release values from WMO (2011). Thus, in our uncertainty analysis for EESC, only the Laube et al. (2013) fractional release uncertainty percentages have been used, not the absolute fractional release values. However, the fractional release values from Laube et al. (2013) have been applied for sensitivity analyses in the calculation of ODPs (Sect. 5).

Following the approach taken in past ozone assessments, two quantities are derived from the EESC time series, namely, the year EESC returns to a pre-1980 level and the integrated EESC above the 1980 level. These quantities have been used extensively to compare different ODS scenarios (WMO, 2003, 2007, 2011).

\subsection{ODS production, banks, and observed mixing ratios}

We use production data from 1986 to 2008 that have been officially reported by countries to UNEP. For the production from 2009 and after, the data from the baseline scenario of WMO (Daniel and Velders, 2011) is used. Apart from being used as feedstock, there is no known remaining production of $\mathrm{CCl}_{4}$ for its historical primary uses (e.g., as a solvent) and also no corresponding bank, but there are still considerable emissions as derived from observed mixing ratios. Therefore, following WMO (2011), the relative annual change in emission from 2004 to 2008 is extrapolated from 2009 to 2050. As a consequence of this approach, these future emissions depend on the lifetime of $\mathrm{CCl}_{4}$ since it is a key factor for deriving its top-down emissions over the last few years. This uncertainty in the source of current $\mathrm{CCl}_{4}$ emissions also suggests that future $\mathrm{CCl}_{4}$ emissions are more uncertain than emissions for most of the other ODSs; because it is not clear how to quantify this extrapolation uncertainty, it is not included in our uncertainty analysis.

In addition to the baseline scenario, a zero emissions (from 2014 onward) scenario (see also Daniel and Velders, 2011)) is used to put the results in perspective of the maximum mitigation that could be accomplished for ODSs, barring active removal from the atmosphere.

As described in Sect. 2.1 the bank of ODSs for the year 2008 is used as a constraint for the future calculations with the model. These bottom-up estimates are derived from inventories of the number of units of equipment that use a particular ODS and the amount of ODS in each unit. The bank sizes used here are those used in WMO (2011) and reported by IPCC/TEAP (2009).

Another constraint for the model calculations is the observed surface mixing ratios, which are used to derive topdown emission estimates and as a starting point for the mixing ratio projections. The observations up to the beginning of 2009 are from WMO (2011) for all ODSs. For most species they are based on the average of the annual global mean values from the AGAGE (Advanced Global Atmospheric Gases Experiment) and NOAA/ESRL (National Oceanic and Atmospheric Administration/Earth System Research Laboratory) networks.

\subsection{Lifetimes of ODSs}

The lifetimes of ODSs can be estimated from their loss rates and play an important role in the model calculations. A number of the ODSs - all CFCs, $\mathrm{CCl}_{4}$ and halon-1301 - are removed primarily by photolysis in the stratosphere (SPARC, 2013). The lifetimes of these ODSs depend on the absorption cross sections of the ODSs and solar irradiances, which partially depend on oxygen and ozone opacities. The dependence on the solar irradiances and opacities means that the uncertainties in these ODS photolysis rates, and consequently the lifetimes, are strongly correlated.

Other ODSs - all HCFCs, $\mathrm{CH}_{3} \mathrm{CCl}_{3}$, halon-1211, -1202 and $-2402, \mathrm{CH}_{3} \mathrm{Br}$ and $\mathrm{CH}_{3} \mathrm{Cl}$ - are removed primarily in the troposphere by reaction with the hydroxyl radical $(\mathrm{OH})$, although a significant fraction of halon-1211 and halon-2402 is removed in the stratosphere (SPARC, 2013). Therefore, the uncertainty in the lifetime of these ODSs depends on the uncertainty in the $\mathrm{OH}$ radical abundance in the troposphere. The uncertainties of these ODS lifetimes are therefore also strongly correlated.

In the model calculations the total steady-state lifetimes as reported by WMO (2011) are used as well as those reported by SPARC (2013). The effect of the differences in lifetimes on future mixing ratios and EESC is examined (Sect. 3.1).

Simulations with several 2-D and 3-D atmospheric chemistry-transport models reported by SPARC (2013) show instantaneous lifetimes from 1960 to 2010 that deviate somewhat from the steady-state lifetimes. Instantaneous lifetimes of most ODSs decrease from 1960 to 2010, most 
Table 1. Lifetimes and uncertainties of ODSs from WMO (2011) and SPARC (2013).

\begin{tabular}{lccll}
\hline & \multicolumn{2}{c}{ Steady-state lifetime $(\mathrm{yr})$} & \multicolumn{2}{c}{ Uncertainty in lifetime $(1 \sigma)^{\mathrm{c}}$} \\
\hline & WMO $(2011)$ & SPARC $(2013)$ & Possible & Most likely \\
$\mathrm{CFC}-11$ & 45 & 52 & $\pm 22 \%$ & $\pm 11 \%$ \\
$\mathrm{CFC}-12$ & 100 & 102 & $\pm 15 \%$ & $\pm 8 \%$ \\
$\mathrm{CFC}-113$ & 85 & 93 & $\pm 17 \%$ & $\pm 7 \%$ \\
$\mathrm{CFC}-114$ & 190 & 189 & $\pm 12 \%$ & \\
$\mathrm{CFC}-115$ & 1020 & 540 & $\pm 17 \%$ & \\
$\mathrm{CCl}_{4}$ & $26^{\mathrm{a}}$ & $30^{\mathrm{a}}$ & $\pm 17 \%$ & $\pm 12 \%$ \\
$\mathrm{CH}_{3} \mathrm{CCl}$ & $5.0^{\mathrm{a}}$ & $4.8^{\mathrm{a}}$ & $\pm 3 \%$ & \\
$\mathrm{HCFC}_{3} 22$ & 11.9 & 12 & $\pm 16 \%$ & \\
$\mathrm{HCFC}-141 \mathrm{~b}$ & 9.2 & 9.4 & $\pm 15 \%$ & \\
$\mathrm{HCFC}-142 \mathrm{~b}$ & 17.2 & 18 & $\pm 14 \%$ & \\
$\mathrm{Halon}-1211$ & 16 & 16 & $\pm 29 \%$ & \\
$\mathrm{Halon}-1202$ & 2.9 & 2.5 & $\pm 33 \%$ & \\
$\mathrm{Halon}-1301$ & 65 & 72 & $\pm 13 \%$ & $\pm 9 \%$ \\
$\mathrm{Halon}-2402$ & 20 & 28 & $\pm 19 \%$ & \\
$\mathrm{CH}{ }_{3} \mathrm{Br}$ & $0.75^{\mathrm{a}} \mathrm{b}$ & $0.7^{\mathrm{a}}$ & $\pm 17 \%$ & \\
$\mathrm{CH}{ }_{3} \mathrm{Cl}$ & $1.0^{\mathrm{a}}$ & $0.9^{\mathrm{a}}$ & $\pm 18 \%$ & \\
\hline
\end{tabular}

a Losses due to oceanic and soil processes are taken into account using values from WMO (2011). The partial lifetime for $\mathrm{CCl}_{4}$ is $44 \mathrm{yr}$ for atmospheric loss and $94 \mathrm{yr}$ for oceanic loss (Yvon-Lewis and Butler, 2002). The partial lifetime for $\mathrm{CH}_{3} \mathrm{CCl}_{3}$ is $5.0 \mathrm{yr}$ for atmospheric loss and $94 \mathrm{yr}$ for oceanic loss. The partial lifetime for $\mathrm{CH}_{3} \mathrm{Br}$ is $1.5 \mathrm{yr}$ for atmospheric loss, 2.2-2.4 yr for oceanic loss, and 3.3-3.4 yr for soil loss. The partial lifetime for $\mathrm{CH}_{3} \mathrm{Cl}$ is $1.3 \mathrm{yr}$ for atmospheric loss and $3 \mathrm{yr}$ for oceanic and soil loss.

${ }^{\mathrm{b}}$ In WMO (2011) a best-estimate lifetime for $\mathrm{CH}_{3} \mathrm{Br}$ of $0.8 \mathrm{yr}$ is reported, but in the scenario calculations a value of $0.75 \mathrm{yr}$ is used to be consistent with earlier emission estimates.

${ }^{c}$ Uncertainty in only the atmospheric loss rate (inverse of the lifetime) from SPARC (2013) is taken into account. This is relevant for $\mathrm{CCl}_{4}$, for which the uncertainty could change if the uncertainty in the partial lifetime due to oceanic loss (82-191 yr; WMO, 2011) were to be taken into account.

dramatically in the early years of their use, mainly as a result of large fractional trends in atmospheric concentrations that lead to strong changes in relative mixing ratios in regions close to emissions compared with regions close to losses (Martinerie et al., 2009). The model calculations show only a small trend in lifetimes from 2010 to 2100 . These time-varying instantaneous lifetimes have not been used in the calculations here, since their effects on mixing ratios or inverse-derived emissions are relatively small. The instantaneous lifetimes of CFC-11 and CFC-12 are about $50 \%$ larger than the steady-state lifetimes around 1960, with the difference decreasing to about $20 \%$ around 1980 and becoming even smaller towards 2010. Annual emissions of CFC11 and $\mathrm{CFC}-12$ derived from observed mixing ratios using these instantaneous lifetimes are only 0-4\% smaller over the period 1960-2010 than those derived using the steady-state lifetimes.

\subsection{Parameters for Monte Carlo uncertainty analysis}

A Monte Carlo analysis is used to estimate the total uncertainty in EESC and the estimated time to return to pre1980 EESC levels. It this analysis, uncertainties are applied to all relevant parameters, including (1) annual historic and future production, (2) bank sizes of 2008, (3) observed mixing ratios, (4) fraction of the bank emitted annually $\left(E_{\mathrm{f}}\right),(5)$ loss rate (inverse of the lifetime) of the ODSs, (6) fractional release values, (7) the factor $(\alpha)$ for bromine efficiency in ozone depletion, (8) mean age of air, and (9) the factor relating the surface mixing ratio to the average atmospheric mixing ratio $\left(F_{\text {surf }}\right)$. In calculating the radiative forcing and GWPs of ODSs an additional uncertainty in the radiative efficiency is taken into account. For $\alpha$ and the mean age of air, different values are used for midlatitude and Antarctic conditions. See Tables 1 and 2 for an overview of all the uncertainties used. In SPARC (2013) two uncertainty ranges are given for the ODS loss rate and lifetime, a "possible range" and a "most likely" range. These ranges differ among ODSs for which different methods have been used to derive the loss rate, such as modeling with atmospheric chemistry-transport models, inverse modeling using observed mixing ratios, analysis of satellite data, or tracer-tracer studies. The "possible range" is derived from the joint distribution of the different methods and is likely an overestimation of the true uncertainty, since it reflects the full range in the lifetime estimates. The "most likely range" is derived from the combined distribution of the different methods and represents the variability of the best estimate. This range is likely an underestimation of the true uncertainty since, for example, it assumes the different methods are uncorrelated and that they are estimating the same quantity. Both uncertainty ranges are presented and discussed in our uncertainty analysis because the uncertainty 
Table 2. Parameters and uncertainties used in the model calculations.

\begin{tabular}{|c|c|c|c|}
\hline & Value & Uncertainty $(1 \sigma)$ & Note \\
\hline Lifetimes through oxygen photolysis & see Table 1 & see Table $1, \gamma=0.9$ & (d) \\
\hline Lifetimes through $\mathrm{OH}$ abundance & see Table 1 & see Table $1, \gamma=0.9$ & (e) \\
\hline Production data & WMO (2011) & $\pm 5 \%$ & (f) \\
\hline Bank size of 2008 & WMO (2011)(a) & $\pm 10 \%$ for CFC- 12, CFC- 115, HCFC $-22, \pm 20 \%$ for other ODSs & $(\mathrm{g})$ \\
\hline Emission factor $\left(E_{f}\right)$ & Calculated & $\pm 10 \%$ for CFC-12, CFC- 115, HCFC- $22, \pm 20 \%$ for other ODSs & (g) \\
\hline Alpha $(\alpha)$ midlatitudes & 60 & $\pm 25 \%$ & $(h, i)$ \\
\hline Alpha $(\alpha)$ Antarctic & 65 & $\pm 25 \%$ & $(h, i)$ \\
\hline Mean age of air, midlatitudes & $3.0 \mathrm{yr}$ & $\pm 0.3 \mathrm{yr}$ & (i) \\
\hline Mean age of air, Antarctic & $5.5 \mathrm{yr}$ & $\pm 0.3 \mathrm{yr}$ & (i) \\
\hline Fractional release & Newman et al. (2006)(b) & $\pm 20 \%$ for $\mathrm{HCFCs}, \pm 10 \%$ for other ODSs & (j) \\
\hline Observed mixing ratios & - & $\pm 0.1 \mathrm{ppt}, \gamma=0.9$ & $(\mathrm{k})$ \\
\hline Surface factor $\left(F_{\text {surf }}\right)$ & $1.07(\mathrm{c})$ & $\pm 3 \%$ & (1) \\
\hline Radiative efficiency & WMO (2011) & $\pm 5 \%$ & $(\mathrm{~m})$ \\
\hline Absolute GWP of $\mathrm{CO}_{2}$ & IPCC (2007) & $\pm 9 \%, \pm 13 \%, \pm 15 \%$ & (n) \\
\hline
\end{tabular}

(a) The sizes of the banks in 2008 as used in WMO (2011). The following bank sizes are from bottom-up analysis of TEAP (2009): 1,420 kt for CFC-11, 394 kt for CFC-12, 16 kt for

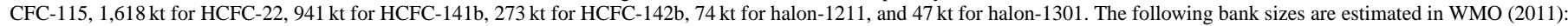
$25 \mathrm{kt}$ for CFC-113, $15 \mathrm{kt}$ for CFC-114, and $11 \mathrm{kt}$ for halon-2402.

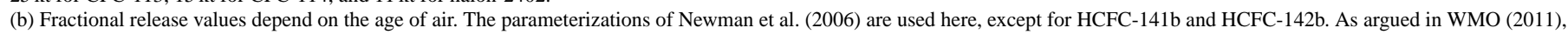

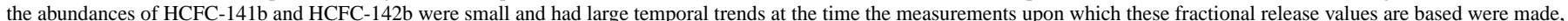

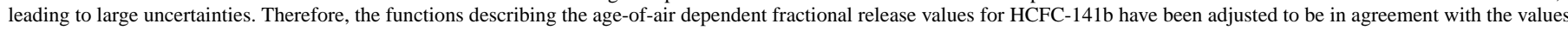

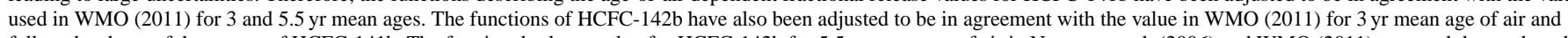

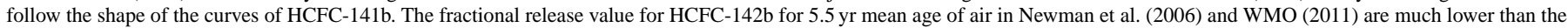

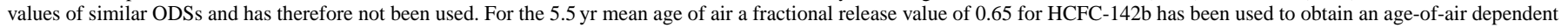

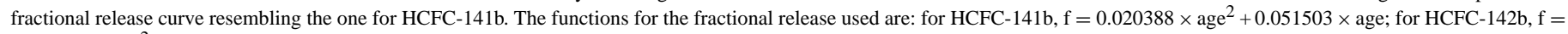
$0.024739 \times \mathrm{age}^{2}-0.017885 \times$ age

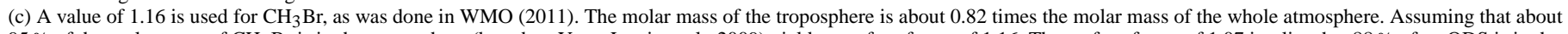

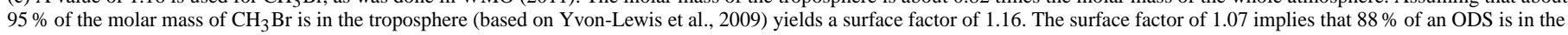
troposphere.

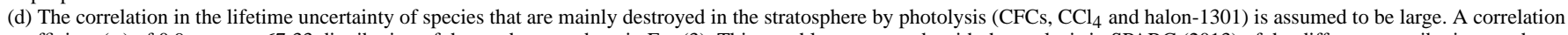
coefficient $(\gamma)$ of 0.9 means a 67:33 distribution of the random numbers in Eq. (3). This roughly corresponds with the analysis in SPARC (2013) of the different contributions to the uncertainty in the total ODS lifetime.

(e) The correlation in uncertainty in lifetimes of species that are mainly destroyed in the troposphere by reaction with $\mathrm{OH}\left(\mathrm{HCFCs}, \mathrm{CH}_{3} \mathrm{CCl}\right.$, halon-1211, halon- 1202 , halon-2402,

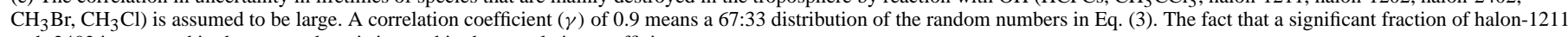
and -2402 is removed in the stratosphere is ignored in the correlation coefficient.

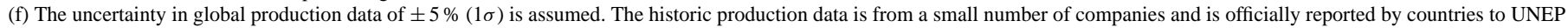
(2010).

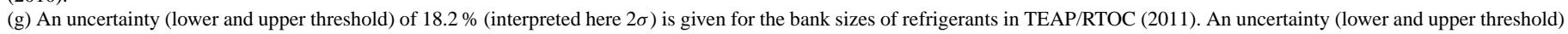

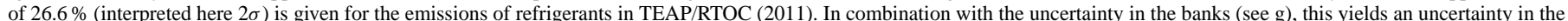

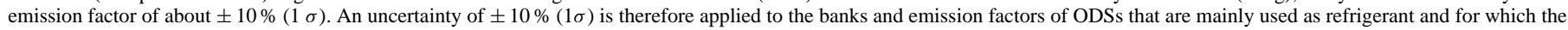

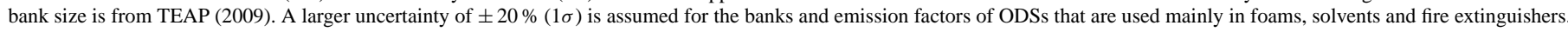
(h) Alpha $(\alpha)$ value from WMO (2011).

(i) Uncertainty from Newman et al. (2007)

(j) Uncertainty from Laube et al. (2013).

(k) An uncertainty if the global and annual average observed mixing ratio of $\pm 0.1 \mathrm{ppt}(1 \sigma)$ is assumed with time correlation coefficient $(\gamma)$ of 0.9 .

(l) An uncertainty of $\pm 3 \%(1 \sigma)$ is assumed following Daniel and Velders (2007).

(m) An uncertainty of $\pm 5 \%(1 \sigma)$ is used, based on a reported uncertainty of $\pm 10 \%$ (assumed to be $2 \sigma$ ) by IPCC (2001).

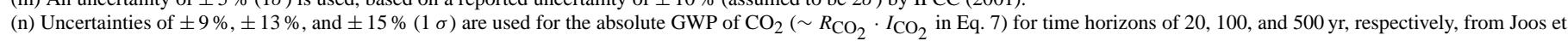
al. (2013) and IPCC (2013).

in the lifetimes is the most important parameter in the analysis.

As mentioned in Sect. 2.3 the loss rates of the ODSs that are removed mainly in the stratosphere all depend on the oxygen and ozone opacity and solar irradiances. As a consequence, the uncertainty in the loss rates of these species is correlated. This correlation is taken into account in the Monte Carlo analysis by applying two random numbers to determine the loss rate of an ODS. A random variation of the loss rate of ODS $i$, denoted by $\sigma_{r}\left(L_{i}\right)$, is calculated using Eq. (3),
$\sigma_{r}\left(L_{i}\right)=\sigma\left(L_{i}\right) \cdot\left(\operatorname{random}(1) \cdot \gamma+\operatorname{random}(2) \cdot \sqrt{1-\gamma^{2}}\right)$,

where $\sigma\left(L_{i}\right)$ is the uncertainty in the loss rate and "ran$\operatorname{dom}()$ " indicates a random number drawn from a normal distribution $(\mathrm{N}(0,1))$. Correlation between loss rates $\sigma_{r}\left(L_{i}\right)$ of different ODSs is obtained by applying the same random number (random (1)) to all ODSs that are destroyed mainly by photolysis in the stratosphere. A default correlation coefficient $\gamma$ of 0.9 is assumed here. Such a coefficient means that $67 \%$ of the random variation of the uncertainty in the loss rate of an ODSs in Eq. (3) is associated with common uncertainties (i.e., solar irradiance and opacity for species mainly removed in the stratosphere) and $33 \%$ with 
the specific ODSs. This roughly corresponds with the analysis in SPARC (2013) of the different contributions to the uncertainty in the total ODS lifetime. The sensitivity of EESC to the value of $\gamma$ is studied in Sect. 3.2.

The same approach is used for the ODSs that are mainly removed in the troposphere by reaction with the $\mathrm{OH}$ radical. The uncertainties in the loss rates of these species is also correlated using Eq. (3) with the same correlation coefficient, but with different random numbers so that the $\mathrm{OH}-\mathrm{loss}$ uncertainties are not correlated with the photolysis loss uncertainties.

The fractional release values depend on the age of air following Newman et al. (2006). In addition to the uncertainty in the mean age of air an additional uncertainty of \pm 10 to $\pm 20 \%(1 \sigma)$ is applied to the fractional release values of all ODSs (Table 2, Laube et al., 2013).

Uncertainty in global production data is assumed to be small. The historic production data is reported by a small number of companies and officially reported by countries to UNEP (UNEP, 2010). The production data used in the model is only the anthropogenic production. Natural emissions of $\mathrm{CH}_{3} \mathrm{Br}$ and $\mathrm{CH}_{3} \mathrm{Cl}$ are derived from the constant background mixing ratios and the lifetimes. An uncertainty of $\pm 5 \%(1 \sigma)$ is assumed in the annual historic and future production data. It is assumed that the data is $100 \%$ correlated between successive years, but completely uncorrelated among species. In the model, the uncertainty in historical production data only affects future mixing ratios through the emission factors.

The 2008 bank sizes of CFC-11, CFC-12, CFC115, HCFC-22, HCFC-141b, HCFC-142b, halon-1211, and halon-1301 are from TEAP (2009). Banks for CFC-113, CFC-114 and halon-2402 are taken from estimates in WMO (2011). For the other ODSs, $\mathrm{CCl}_{4}, \mathrm{CH}_{3} \mathrm{CCl}_{3}$, halon$1202, \mathrm{CH}_{3} \mathrm{Br}$, and $\mathrm{CH}_{3} \mathrm{Cl}$, banks are expected to be small, so it is assumed that emissions are equal to production and that the bank is thus zero. The fraction of the bank emitted annually is calculated in the model from emissions derived from observed mixing ratios and bank sizes averaged over $10 \mathrm{yr}$ (1999-2008). Based on an uncertainty analysis from TEAP/RTOC (2011), an uncertainty of $\pm 10 \%(1 \sigma)$ is applied to the bank sizes of 2008 for all ODSs used mainly as refrigerants and an uncertainty of $\pm 20 \%(1 \sigma)$ is applied to the bank sizes of the other ODSs. These uncertainties also affect the emission factors, since these factors are calculated as the fraction of the annual emission divided by the bank. In addition, an extra uncertainty of $\pm 10 \%$ is applied to the emission factors of all ODSs used mainly as refrigerants (UNEP, 2011) and $\pm 20 \%$ for the other ODSs. In the Monte Carlo simulations the emission factor is limited in range from 0 to 1 . Uncertainties in bank sizes are assumed to be uncorrelated. Calculations show that future mixing ratios and EESC levels are not very sensitive to the exact magnitude of the uncertainty in the banks (see Sect. 7).

An uncertainty is also applied to the factor $\left(F_{\text {surf }}\right)$, which relates the surface mixing ratio to the corresponding aver- age atmospheric mixing ratio. A value of 1.07 is used for all species, except $\mathrm{CH}_{3} \mathrm{Br}$. For $\mathrm{CH}_{3} \mathrm{Br}$ a value of 1.16 is used (see Table 2). An uncertainty of $\pm 3 \%(1 \sigma)$ is assumed for $F_{\text {surf }}$ for all species, and it is assumed to be $100 \%$ correlated among species.

The uncertainties in the mean age of air and the factor $\alpha$ are from Newman et al. (2007). In the Monte Carlo simulations, the fractional release value is limited in range from 0 to 1 even if the error bars extended past these limits to unphysical values.

Probably the most accurately known quantities used in the model are the observed mixing ratios, taken from WMO (2011). The global and annual average mixing ratios are based on observations by the AGAGE (Prinn et al., 2000) and NOAA networks (Montzka et al., 1999). In the model, the mixing ratios are used to derive top-down emissions and as starting point for the calculation of future mixing ratios. An uncertainty of $\pm 0.1 \mathrm{ppt}(1 \sigma)$ is assumed for the annual average values. Since the observations are always made in the same way, they should generally be correlated in time. A time correlation of 0.9 is therefore assumed between successive years. As is shown in Sect. 3.3 the effect of the uncertainty in the observed mixing ratios on future EESC levels is small, so this assumption about the magnitude of the uncertainty and the temporal correlation is not very important compared with other uncertainties.

If a top-down derived emission for a year becomes negative in a Monte Carlo simulation it is set to zero. This artificially adds emissions to the system and thereby affects the model results, but simulations show that negative emissions occur seldom (less than $0.02 \%$ of the time for most ODSs, but $0.4 \%$ of the time for CFC-113).

Monte Carlo runs are performed with Latin hypercube sampling (McKay et al., 1979), which reduces the number of model evaluations significantly, without compromising the accuracy of the results. The 2.5, 50 and 97.5 percentiles are calculated for the production, bank sizes, emissions, mixing ratios, and emission fraction for each species; these same percentile statistics are also calculated for EESC, radiative forcing, and ODP- and GWP-weighted emissions. Uncertainties are presented for the $95 \%$ confidence interval. The same statistics are calculated for the year EESC returns to pre-1980 levels and the integrated EESC above the 1980 level for midlatitude and Antarctic conditions.

\section{Uncertainties in mixing ratios and EESC}

We will first focus on the effects of the new lifetimes from SPARC (2013) on mixing ratios and EESC compared with results obtained using the lifetimes from WMO (2011). Then the effects of uncertainties in lifetimes and other parameters on mixing ratios and EESC will be discussed, followed by the effects of the uncertainties on the radiative forcing of the ODSs and on ODPs and GWPs. 


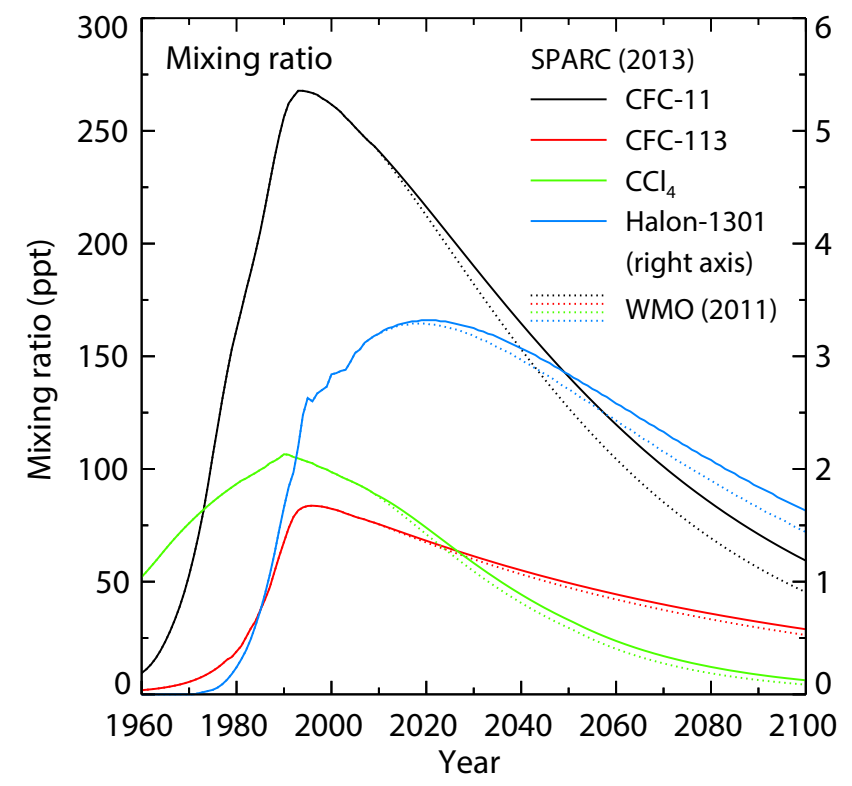

Fig. 1. Mixing ratios based on lifetimes from SPARC (2013) and WMO (2011). The mixing ratios are shown for the ODSs with the most relevant differences in lifetimes for EESC. Before 2010, concentrations are constrained by observations or are fixed from some other information and so the lifetime differences affect calculated emissions but not concentrations.

\subsection{Lifetimes from SPARC versus WMO}

For EESC, the most significant differences in lifetimes between SPARC (2013) and WMO (2011) are for CFC-11 $(+16 \%), \mathrm{CCl}_{4}(+15 \%)$, halon-1301 (+11\%) and CFC$113(+9 \%)$. For these species the newer SPARC (2013) stratospheric lifetimes are all larger than the older ones from WMO (2011). Other differences are for CFC-115 $(-47 \%)$, halon-1202 (-14\%), halon-2402 (+40\%), and $\mathrm{CH}_{3} \mathrm{Cl}(-10 \%)$, but the contribution from anthropogenic emissions of these species to the EESC levels is small (currently about $2 \%$ of total EESC). The mixing ratios of the ODSs based on both sets of lifetimes from 1960 to 2100 are shown in Fig. 1. Using the lifetimes from SPARC (2013) instead of those from WMO (2011) results in higher mixing ratios: $14 \mathrm{ppt}(11 \%)$ for $\mathrm{CFC}-11$ by $2050,3.5 \mathrm{ppt}(12 \%)$ for $\mathrm{CCl}_{4}, 0.1 \mathrm{ppt}(5 \%)$ for halon-1301, $2.1 \mathrm{ppt}(4 \%)$ for CFC$113,1.2 \mathrm{ppt}$ for HCFC-22 (2\%), and $3.0 \mathrm{ppt}$ for CFC-12 $(0.8 \%)$. The higher mixing ratio of $\mathrm{CCl}_{4}$ is a result of a slower decrease of the atmospheric burden because of the lower loss rate (longer lifetime), which is in part (about a third) compensated by the smaller future emissions. As discussed in Sect. 2.2 the future emissions of $\mathrm{CCl}_{4}$ are based on an extrapolation of historic emissions derived from observed mixing ratios and therefore depend on its lifetime.

The effects of the SPARC (2013) versus WMO (2011) lifetimes on the EESC levels for midlatitude and Antarctic conditions are shown in Fig. 2. The EESC levels based on the SPARC (2013) lifetimes are only slightly higher than those based on WMO (2011). The year EESC returns to pre1980 levels for midlatitude conditions is 2046 based on lifetimes from WMO (2011) and about 2 yr later, 2048, based on lifetimes from SPARC (2013). The corresponding years for Antarctic spring conditions are 2071 for WMO (2011) and about 4 yr later, 2075, for SPARC (2013). The change in integrated EESC above the 1980 level is also small, about $2 \%$ for midlatitude and about $3 \%$ for Antarctic conditions. The values calculated here with the lifetimes from WMO (2011) differ slightly from those reported in WMO (2011) because of the use of the age-of-air spectrum in the calculations instead of a single mean age of air.

\subsection{Effects of uncertainties in lifetimes}

Apart from a new set of lifetimes, the SPARC assessment also reported uncertainty ranges for these lifetimes. The effects of these uncertainties on the mixing ratios are shown in Fig. 3. It is clear that the uncertainty ranges yield much larger variations in future mixing ratios than do the absolute differences in lifetimes between WMO (2011) and SPARC (2013). For example, the uncertainty in the lifetimes yields a range in mixing ratio of CFC-11 of about $\pm 35 \mathrm{ppt}$ for the possible range in lifetime and $\pm 19 \mathrm{ppt}$ for the most likely range by 2050 , compared with a difference of 14 ppt resulting from the absolute difference in the lifetime of CFC-11 between SPARC (2013) and WMO (2011). For CFC-12, where there is almost no effect from the difference in lifetime between SPARC (2013) and WMO (2011), and the difference in mixing ratio is $3 \mathrm{ppt}$ in 2050 , the mixing ratio uncertainty is about \pm 48 ppt and about \pm 24 ppt in 2050 for the possible and most likely ranges, respectively, in lifetime.

The effects of the ODS lifetime uncertainties on EESC levels is shown in Fig. 4, while in Fig. 5 (values presented in Table S1 in the Supplement) the effect of the lifetime uncertainty of each individual ODSs on the year EESC returns to pre-1980 levels is presented. In the baseline scenario, without taking uncertainties into account, EESC returns to pre-1980 levels in 2048 for midlatitude conditions and in 2075 for Antarctic spring conditions. The individual ODS that are responsible for the largest uncertainty in the year EESC returns to pre-1980 levels are CFC-11 and halon-1211, followed by CFC-12, $\mathrm{CCl}_{4}$, and $\mathrm{CH}_{3} \mathrm{Br}$. For Antarctic conditions CFC113 and halon-1301 are also important due to their relatively long lifetimes. If uncertainties in lifetimes are applied to several ODSs simultaneously, the correlations between uncertainties become important. As previously stated, correlation is applied to two groups, ODSs that are mainly destroyed by photolysis in the stratosphere and ODSs that are mainly destroyed by reactions with the $\mathrm{OH}$ radical in the troposphere. Without correlation the total uncertainty range in EESC and in the year EESC returns to pre-1980 levels is only slightly larger than the largest contribution of the individual ODSs. If the uncertainties are fully correlated the uncertainty range 

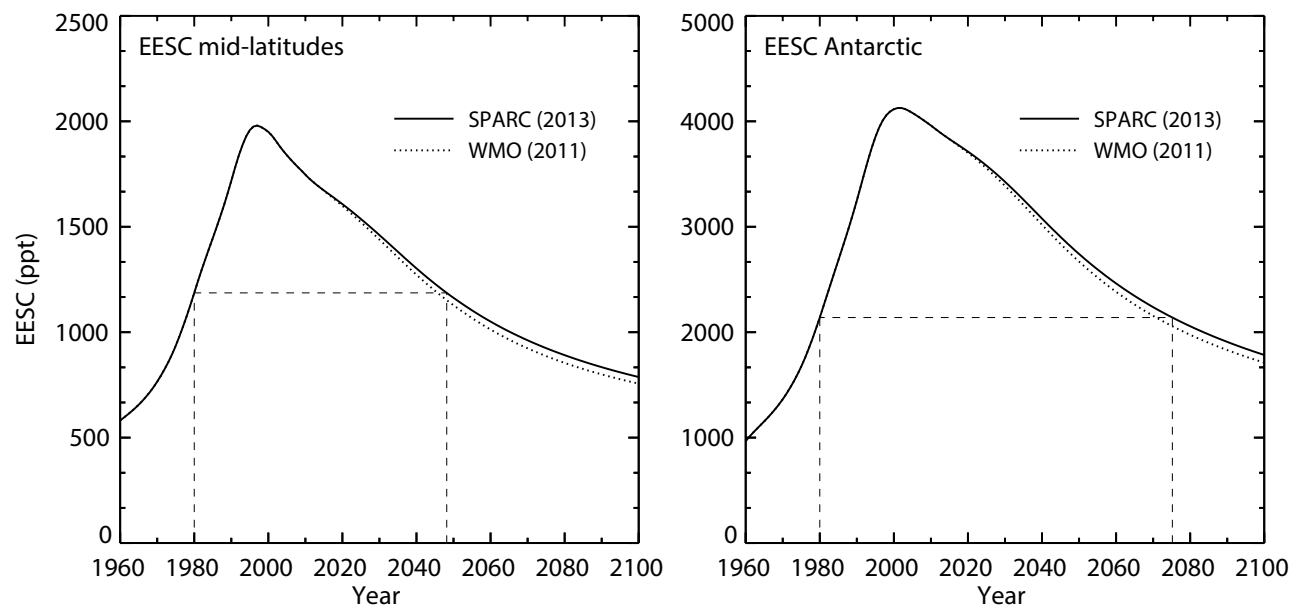

Fig. 2. EESC for midlatitude (left) and Antarctic (right) conditions based on lifetimes from SPARC (2013) and WMO (2011). The thin dashed lines indicate the 1980 EESC levels and the years the EESC curves cross these levels for the cases using the SPARC (2013) lifetimes.
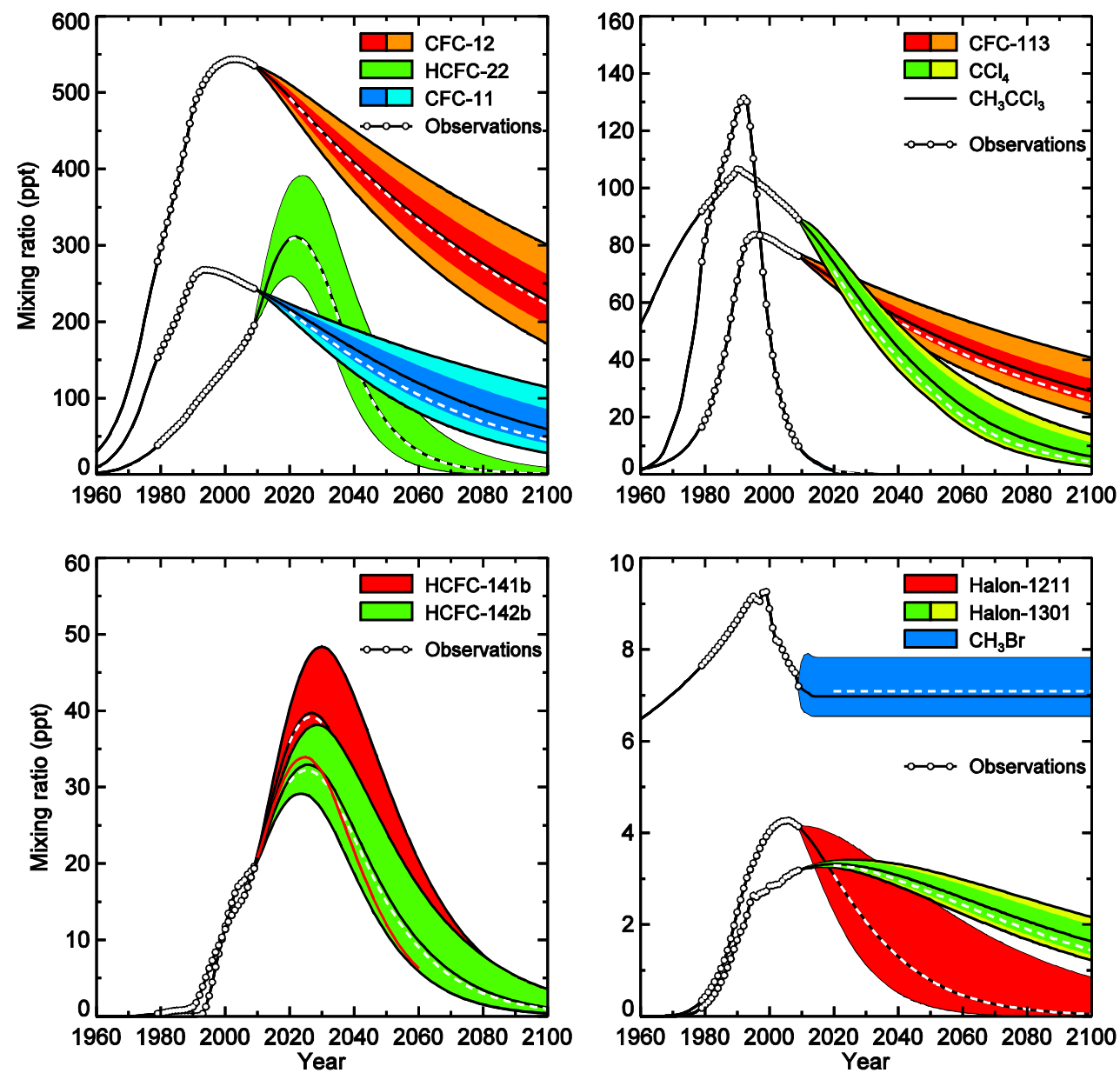

Fig. 3. Mixing ratios of ODSs from 1960 to 2100 based on lifetimes and their uncertainties from SPARC (2013). Shown are the median values and $95 \%$ confidence interval based on the most likely (dark colors) and possible (light colors) uncertainty ranges in lifetimes. Open circles show the observed mixing ratios. White dashed lines represent the median values of the mixing ratios based on lifetimes from WMO (2011). 

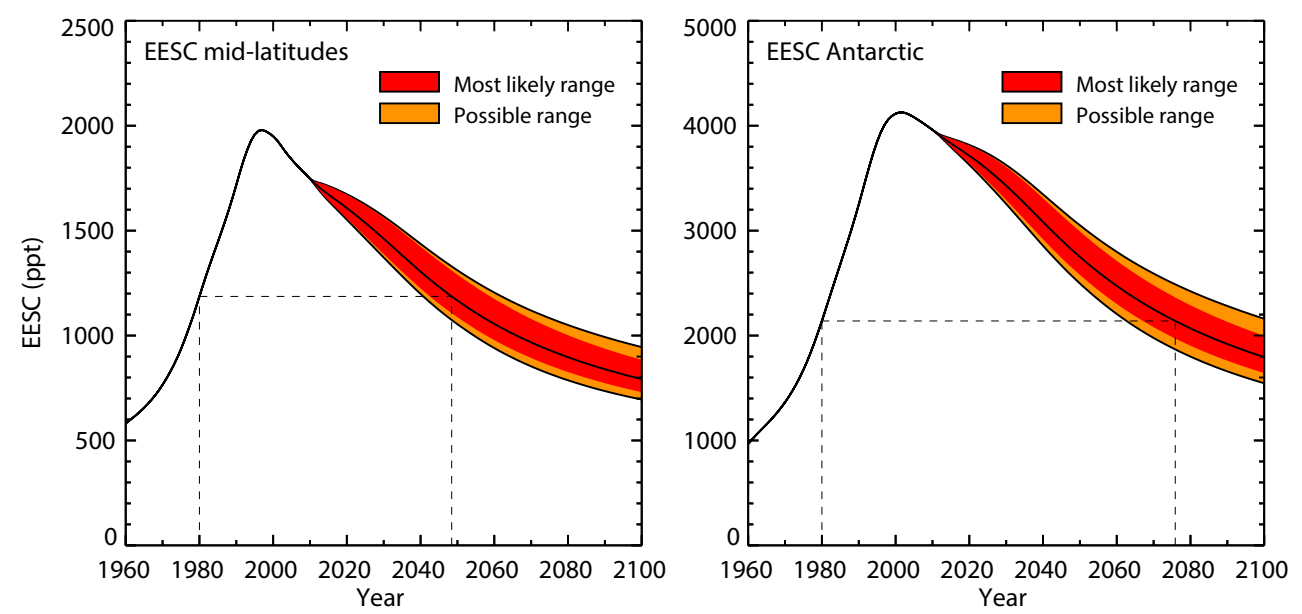

Fig. 4. EESC from 1960 to 2100 for midlatitude (left) and Antarctic (right) conditions using the lifetimes from SPARC (2013) and with uncertainties applied to the loss rates (inverse of lifetimes) of all species, except $\mathrm{CH} 3 \mathrm{Cl}$ and the natural contribution of $\mathrm{CH}_{3} \mathrm{Br}$. $\mathrm{A}$ correlation coefficient $\lambda$ of 0.9 is used for the uncertainties in lifetimes among the ODSs mainly removed in the stratosphere and among those mainly removed in the troposphere. Shown are the median values and $95 \%$ confidence interval based on the possible (orange) and most likely (red) uncertainty ranges in lifetimes. The years EESC return to pre-1980 levels for the median EESC values is indicated with thin dashed lines.
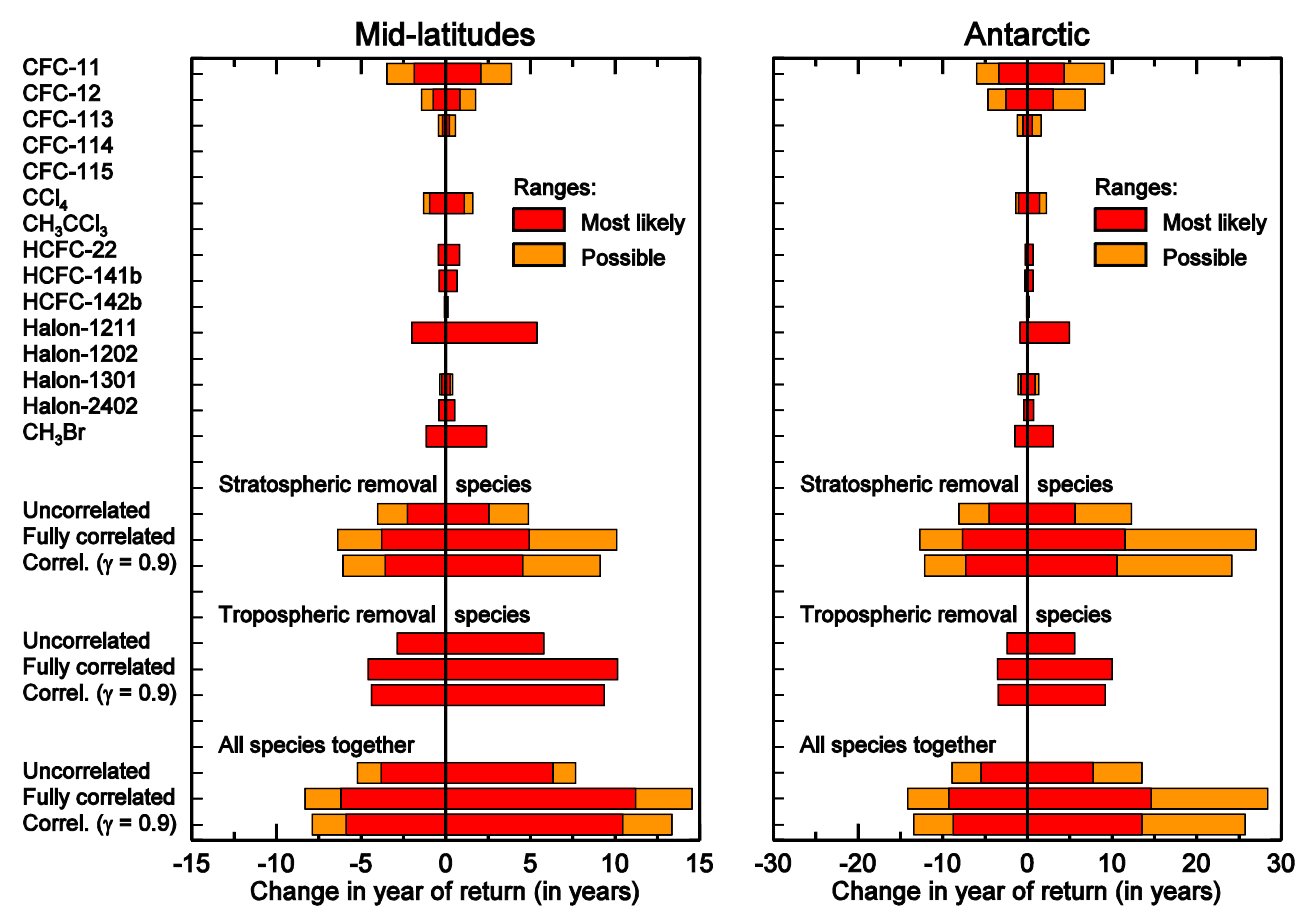

Fig. 5. Uncertainties (difference from the median, $95 \%$ confidence interval) in the years of return to pre-1980 levels for midlatitude and Antarctic springtime conditions resulting from uncertainties in the ODS lifetimes from SPARC (2013). The contributions from the individual ODSs are shown as well as the combined contributions from all species removed primarily in the stratosphere, all removed in the troposphere, and all species together. The combined contributions are shown with uncorrelated uncertainties in lifetimes $(\gamma=0)$, fully correlated uncertainties $(\gamma=1)$, and with a correlation coefficient $\gamma$ of 0.9. The year of return in the base run is 2048 for midlatitudes and 2075 for Antarctic spring conditions. The data of this table is also presented in Table S1 in the Supplement).

in the year of return is approximately twice as large as when fully uncorrelated.

The species mainly removed in the stratosphere and those mainly removed in the troposphere have similar contribu- tions to the total uncertainty in EESC and in terms of year of return to pre-1980 levels for midlatitude conditions (possible range of about -5 to $+9 \mathrm{yr}$ ), with the default correlation coefficient of 0.9. For Antarctic conditions the species mainly 
removed in the stratosphere are the larger contributor to the uncertainty range of -12 to $+24 \mathrm{yr}$ (possible range), due to their longer lifetimes. For those species mainly removed in the troposphere, the possible range equals the most likely range since only one method has been used for deriving the lifetime and the associated uncertainty (SPARC, 2013).

Applying uncertainties to the lifetimes of all ODSs, with a correlation coefficient of 0.9 , yields a possible range in the year of return to pre-1980 levels from -8 to $+13 \mathrm{yr}$ and a most likely range from -6 to $+10 \mathrm{yr}$ for midlatitude conditions. For Antarctic conditions the possible range is from 13 to $+26 \mathrm{yr}$ and the most likely range is from -9 to $+14 \mathrm{yr}$.

The uncertainty in the lifetime of $\mathrm{CH}_{3} \mathrm{Cl}$ is not included in the uncertainty analysis since the emissions of $\mathrm{CH}_{3} \mathrm{Cl}$ are derived from the observed mixing ratios (and the lifetime) and are assumed to remain constant in the scenario from 2009 onward.

\subsection{Overall uncertainty analysis}

Apart from the uncertainties in lifetimes, uncertainties in other parameters also affect future mixing ratios and EESC levels. In Fig. 6 the effect of all uncertainties (Table 2) on the normalized EESC levels from 1960 to 2100 is shown. The EESC levels are normalized (1980 level set to 1) in Fig. 6 to avoid a large range in EESC levels in 1980 resulting from uncertainties in the bromine efficiency factor $\alpha$, in the mean age of air, and in the fractional release values. The uncertainty in the mean age of air represents the uncertainty in the age of air that is considered to be representative for midlatitude and Antarctic conditions. An alternative approach would have been to calculate the EESC for a particular age and ascribe zero uncertainty to that age; however, we have opted for this approach to account for some uncertainty in the choice of the "best" representative age of the midlatitude and Antarctic springtime stratospheres. By normalizing, the absolute differences in EESC due strictly to the magnitude of the fractional release values are removed, but there remain impacts of relative gas-to-gas fractional release changes as well as impacts of the differences in the relative temporal relationship between the tropospheric mixing ratios and EESC at the time of crossing the 1980 level. Also, by normalizing EESC, the year of return to pre-1980 levels can be directly inferred graphically.

The effects of uncertainties of the various parameters on the year EESC returns to pre-1980 levels are shown in Fig. 7 and Table S2. Table S2 also contains data on the integrated EESC above the 1980 level. The largest uncertainty ranges in the year of return are caused by the uncertainties in the lifetimes, followed by the uncertainty in the mean age of air, with smaller contributions from the uncertainties in the fractional release values and bromine efficiency factor $\alpha$. The effects of the uncertainty in the production, bank in 2008, and emission factors is small. One reason these last three factors are of minor importance is that the total amount of projected production and anthropogenic ODSs present in existing applications is much smaller than the total atmospheric burden of these ODSs (see also Sect. 7). Also, the exact rate of release from the existing banks is not critical to determining the year of return to 1980 levels when lifetimes are long. The changes in the projected atmospheric concentrations are strongly affected by the uncertainties in the lifetimes because these directly determine how persistent the various ODSs are in the atmosphere. The uncertainties in the year of return shown in Fig. 7 are in good agreement with those found by Newman et al. (2007). The effects of the uncertainty in the fractional release values discussed here is somewhat larger, but that is caused by the larger assumed uncertainty in these values based on Laube et al. (2013).

Combining all uncertainties, the year EESC returns to pre1980 levels has a possible range of 2039-2064 (-9.8 yr, $+15.8 \mathrm{yr}$ ) and a most likely range of 2040-2061 (-8.6 yr, $+12.7 \mathrm{yr}$ ) for midlatitude conditions. For Antarctic spring conditions the possible range is from 2061 to 2105 ( $-16.1 \mathrm{yr}$, $+28.3 \mathrm{yr}$ ) and the most likely range is from 2064 to 2093 $(-12.8 \mathrm{yr},+16.9 \mathrm{yr})$. The ranges are not very different from those resulting from uncertainties in the lifetimes alone, showing again that the uncertainties in the lifetimes dominate the total uncertainty in the EESC curve's shape over time. The large sensitivity partly results from the small EESC slope around the period of recovery compared with the slope near 1980; however, it should also be noted that because of the slow EESC decay projected for the middle part of this century, the absolute difference in ozone depletion could be rather small and still lead to a large difference in the return time.

To put the results of the uncertainty analysis in perspective they are compared with a zero emissions scenario, a zero production scenario, and a scenario with additional emissions. In a scenario with a cease in production of all (anthropogenic) ODSs starting in 2014, the year EESC returns to pre-1980 levels is 2045 for midlatitudes and 2073 for Antarctic springtime, which is about $3 \mathrm{yr}$ earlier than in the baseline scenario in each case. Similarly, if all anthropogenic ODS emissions were to cease in 2014, the year EESC would return to pre1980 levels is 2037 for midlatitude conditions and 2063 for Antarctic conditions, about $12 \mathrm{yr}$ earlier than in the baseline scenario. So, the lower end of the uncertainty range, about 2039 for midlatitudes and 2061-2064 for Antarctic conditions, corresponds approximately to a zero emissions scenario in terms of return to 1980 EESC levels. With respect to the higher end of the uncertainty range in EESC, this can also be reached by a scenario with the best lifetime estimates but with extra ODS emissions on top of the emissions in the baseline scenario. An additional emission of 4 Mt CFC-11 and $4 \mathrm{Mt}$ CFC-12 in 2015 will increase EESC for midlatitude and Antarctic conditions to such levels that the years EESC return to pre-1980 levels matches the higher end of the uncertainty range. These extra emissions of about $7 \mathrm{Mt}$ CFC-11 eq (eq. - equivalent). correspond with 12 times the 

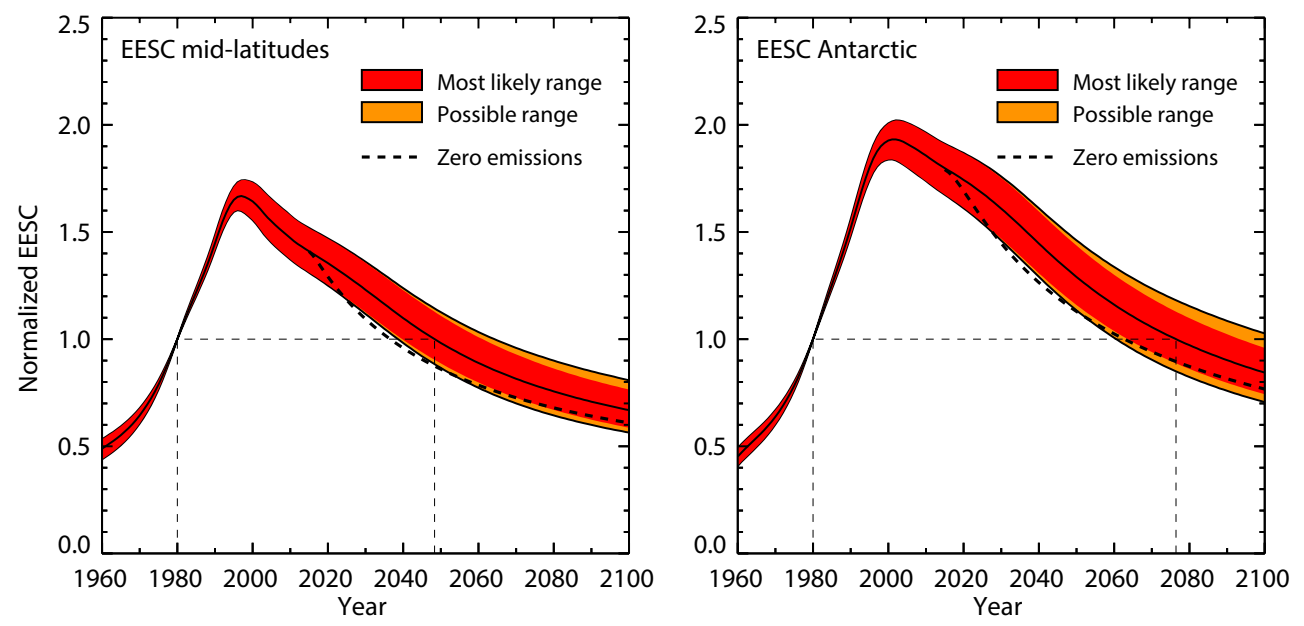

Fig. 6. Normalized EESC (1980 value set to 1) from 1960 to 2100 for midlatitude (left) and Antarctic (right) conditions using the lifetimes from SPARC (2013) and with uncertainties applied to all parameters. Shown are the median values and $95 \%$ confidence interval based on the possible (orange) and most likely (red) uncertainty ranges in lifetimes. The years EESC returns to pre-1980 levels for the median EESC values is indicated with thin dashed lines. The EESC curves corresponding to the zero-emission scenario are shown for reference.
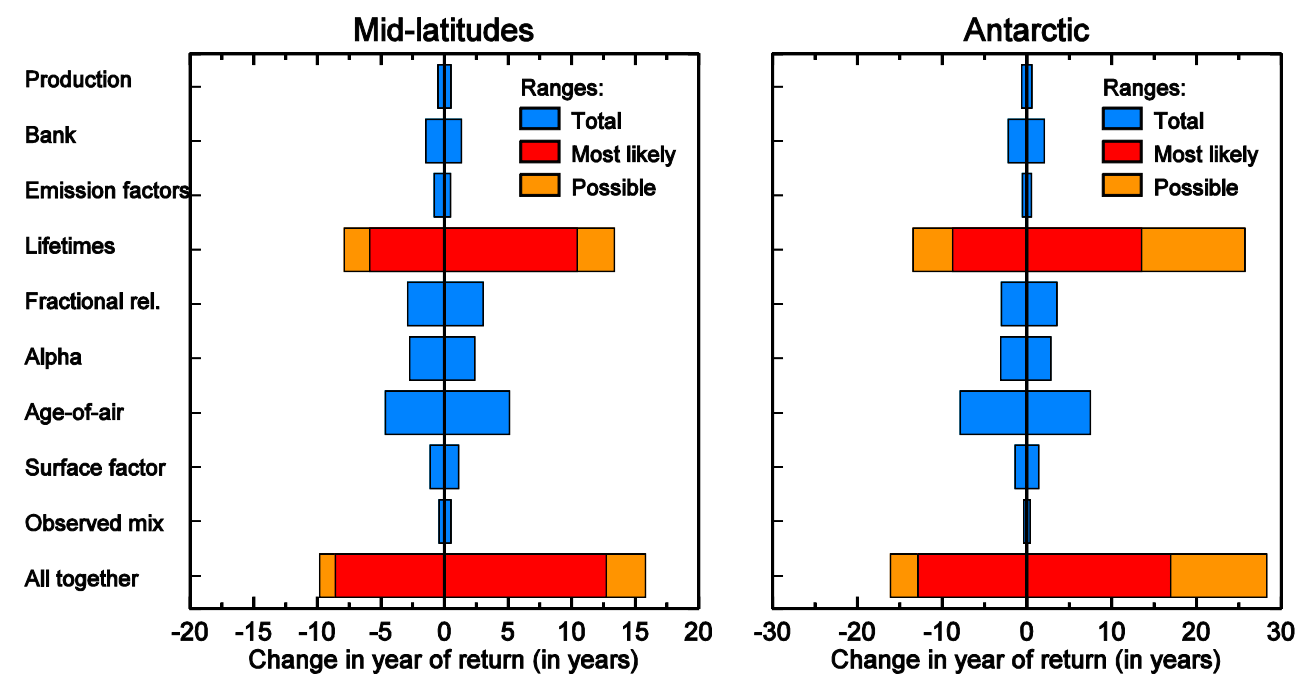

Fig. 7. Uncertainties (difference from the median, $95 \%$ confidence interval) in the years of return to pre-1980 levels for midlatitude and Antarctic springtime conditions resulting from uncertainties in all considered model input parameters. The year of return in the base run is 2048 for midlatitudes and 2075 for Antarctic spring conditions.

cumulative absolute HCFC emissions from 2014 to 2050 or about 2 times the cumulative (anthropogenic) emissions of all ODSs from 2014 to 2050.

The uncertainty analyses also yield confidence intervals for the banks and emissions of each ODS. In Fig. S1 in the Supplement, the effects of applying uncertainties of all parameters on the banks, emissions, and mixing ratios of the main ODSs are shown. The uncertainty ranges in mixing ratios are almost identical to those in Fig. 3, again demonstrating the key role of the lifetime uncertainty. As discussed in Sect. 7 the uncertainty ranges of HCFC-141b, HCFC$142 \mathrm{~b}$, and halon-1301 are also significantly affected by un- certainties in parameters other than the lifetimes. The ODSs with large banks continuing for several decades are CFC-11, HCFC-22, HCFC-141b, and to a lesser extent, the halons, with CFC-11 having the largest uncertainty in the size of the bank. It should be noted, however, that the halon banks are more important to EESC and ozone depletion than their size would suggest due to the bromine efficiency factor, $\alpha$. The uncertainty range in the future emissions is much smaller than in the size of the bank, which is clearly visible, for example, for CFC-11. This different behavior in the size of the bank and future emissions is a result of the way emissions are calculated from the bank and the emission factor. A larger 
size of the bank corresponds with a smaller emission factor, thereby reducing the effect on future emissions (see also Sect. 7).

The year 1980 is used here (Figs. 5, 7) and in several WMO ozone assessments as an EESC reference year for one measure of partial ozone recovery. The choice of this particular year and corresponding EESC level is somewhat arbitrary, since model calculations show that ozone depletion must have occurred before 1980 (WMO, 2011). Using a different year will change the year EESC returns to pre-1980 levels, but will not significantly affect the relative contributions to the uncertainty analysis presented here. For example, the year EESC returns to pre-1975 levels is about three decades later than to pre-1980 levels. The EESC slope is smaller at the time of crossing below the 1975 EESC level, which leads to larger uncertainty magnitudes than in the 1980 return case. However, the lifetime uncertainty remains the most important factor to the overall uncertainty.

In Table S2 in the Supplement the effects of the uncertainties in model parameters on EESC are also compared with respect to the change in integrated EESC above the 1980 level. This is a metric commonly used in WMO assessments for comparing different ODS scenarios. It takes into account the time evolution of the ODSs and not only the year EESC levels drop below the 1980 level.

\section{Uncertainties on radiative forcing of ODSs}

ODSs not only deplete the ozone layer, but they are also strong greenhouse gases (Ramanathan, 1975; Velders et al., 2007). In Fig. 8, the radiative forcing of the ODSs is shown. The uncertainty range in radiative forcing before 2010 is almost completely the result of the applied uncertainty of $\pm 5 \%$ (Table 2) in the radiative efficiency of the ODSs (Table 2). From 2020 onward it is almost completely determined by the uncertainty in the lifetimes of the ODSs. The effect of all other uncertainties on the radiative forcing is very small.

The radiative forcing of the ODSs is about $0.32 \mathrm{~W} \mathrm{~m}^{-2}$ in 2010 (WMO, 2011; Montzka et al., 2011). Taking into account an uncertainty in the radiative efficiency this becomes a range ( $95 \%$ confidence interval) of $0.30-0.34 \mathrm{~W} \mathrm{~m}^{-2}$. The radiative forcing is expected to decrease to $0.20 \mathrm{~W} \mathrm{~m}^{-2}$ $(0.17-0.23)$ in 2050 and to $0.10 \mathrm{~W} \mathrm{~m}^{-2}(0.07-0.14)$ in 2100 (both "possible ranges"). This uncertainty of about \pm $0.035 \mathrm{~W} \mathrm{~m}^{-2}$ in 2100 is larger than the difference between the baseline and zero emissions (2014 onward) scenario of $0.03 \mathrm{~W} \mathrm{~m}^{-2}$ in 2050 and $0.006 \mathrm{~W} \mathrm{~m}^{-2}$ in 2100 . To put the uncertainty range into perspective it can also be compared with the projected radiative forcing from, for example, nitrous oxide $\left(\mathrm{N}_{2} \mathrm{O}\right)$ in the representative concentration pathways (RCPs). In the four RCP scenarios the mixing ratio of $\mathrm{N}_{2} \mathrm{O}$ increases from about $322 \mathrm{ppb}$ in 2009 (Montzka et al., 2011) to 342-367 ppb in 2050 (Meinshausen et al., 2011). This corresponds to an increase in radiative forcing from $0.17 \mathrm{~W} \mathrm{~m}^{-2}$

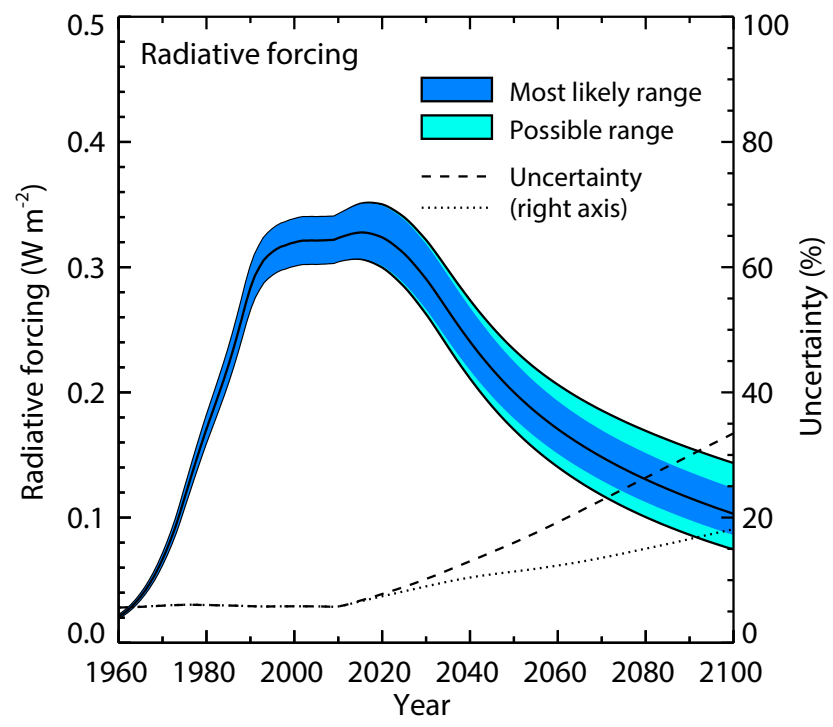

Fig. 8. Radiative forcing of all ODSs from 1960 to 2100 using the lifetimes from SPARC (2013) and with uncertainties applied to all parameters, including the radiative efficiencies. Shown are the median values and $95 \%$ confidence interval based on the possible (light blue) and most likely (dark blue) uncertainty ranges in the lifetime contributions to the total uncertainties. The relative uncertainties (95\% confidence interval) are also shown as percentages of the median for the possible (dashed line) and most likely (dotted line) ranges.

in 2009 to $0.23-0.31 \mathrm{~W} \mathrm{~m}^{-2}$ in 2050 . So even though ODS concentrations are projected to decline substantially over the rest of this century, the uncertainty in their forcing (about $0.035 \mathrm{~W} \mathrm{~m}^{-2}$ ) is significant when compared with $\mathrm{N}_{2} \mathrm{O}$ forcing changes (0.06-0.14 $\mathrm{W} \mathrm{m}^{-2}$ in 2050) and other non- $\mathrm{CO}_{2}$ greenhouse gases. The uncertainty is small compared with the change in forcing from $\mathrm{CO}_{2}$ of $0.7-1.8 \mathrm{~W} \mathrm{~m}^{-2}$ from 2009 to 2050 from the four RCP scenarios (Meinshausen et al., 2011).

\section{Ozone depletion potentials}

A change in the lifetimes of the ODSs also affects ODPs. ODPs are indices used to compare the ability of ODSs to destroy stratospheric ozone relative to that of CFC-11 (Wuebbles, 1983; Fisher et al., 1990). ODPs can be calculated using atmospheric chemistry-transport models, but can also be estimated by a semiempirical approach proposed by Solomon et al. (1992). The semiempirical ODP is defined as

$\mathrm{ODP}_{i}=\left(\alpha n_{\mathrm{Br}, i}+n_{\mathrm{Cl}, i}\right) \frac{f_{i}}{f_{\mathrm{CFC}-11}} \frac{\tau_{i}}{\tau_{\mathrm{CFC}-11}} \frac{M_{\mathrm{CFC}-11}}{M_{i}} \frac{1}{3}$

where $M_{i}$ the molecular weight of species $i\left(\mathrm{~g} \mathrm{~mol}^{-1}\right), f_{i}$ the fractional release value (typically taken for an age of air of $3 \mathrm{yr}$ for midlatitude conditions), $\alpha$ the bromine efficiency factor, $n_{i}$ the number of chlorine or bromine atoms, and $\tau_{i}$ 
the lifetime. The fractional uncertainty is thus defined as the square root of the following:

$$
\begin{aligned}
& \left(\frac{\sigma_{\mathrm{ODP}_{i}}}{\mathrm{ODP}_{i}}\right)^{2} \approx\left(\frac{\sigma_{f_{i}}}{f_{i}}\right)^{2}+\left(\frac{\sigma_{f_{\mathrm{CFC}-11}}}{f_{\mathrm{CFC}-11}}\right)^{2}+\left(\frac{\sigma_{\tau_{i}}}{\tau_{i}}\right)^{2} \\
& +\left(\frac{\sigma_{\tau_{\mathrm{CFC}-11}}}{\tau_{\mathrm{CFC}-11}}\right)^{2}-2 \frac{\sigma_{\tau_{i}}}{\tau_{i}} \frac{\sigma_{\tau_{\mathrm{CFC}-11}}}{\tau_{\mathrm{CFC}-11}} \operatorname{cor}\left(\tau_{i}, \tau_{\mathrm{CFC}-11)}\right. \\
& +\left(\frac{\sigma_{\alpha} n_{\mathrm{Br}, i}}{\alpha n_{\mathrm{Br}, i}+n_{\mathrm{Cl}, i}}\right)^{2}
\end{aligned}
$$

where $\sigma$ is the standard deviation of a quantity and $\operatorname{cor}\left(\tau_{i}, \tau_{\mathrm{CFC}-11}\right)$ is the correlation coefficient between the lifetimes of species $i$ and CFC-11. ODPs calculated by different models and some uncertainties in the calculation of ODPs have been discussed before (Fisher et al., 1990; Solomon and Albritton, 1992; Solomon et al., 1992) but, to our knowledge, an uncertainty analysis as presented here has not been reported.

In Table 3 the steady-state semiempirical ODPs are shown using lifetimes from WMO (2011) and SPARC (2013), the fractional release values from WMO (2011), which are the same as those in Newman et al. (2006) for most species, for a mean age of air in the stratosphere of $3 \mathrm{yr}$, and a value of 60 for the bromine efficiency factor $(\alpha)$ in destroying ozone. Table 3 also contains the uncertainties in the calculated ODPs, based on the uncertainties in the lifetimes, fractional release values for a fixed age of air of $3 \mathrm{yr}$, and factor $\alpha$. The calculated ODPs using the lifetimes from SPARC (2013) are for most species lower, up to $25 \%$, than when using the lifetimes reported in WMO (2011). These differences are mainly the result of the increase of $16 \%$ in the CFC-11 lifetime estimate in SPARC (2013). The two exceptions are the ODPs of CFC115 , which is about $50 \%$ lower, and of halon-2402, which is about $20 \%$ higher than in WMO (2011). The uncertainties (95\% confidence interval) in ODPs are $30-35 \%$ for the CFCs, $55-70 \%$ for the HCFCs, and from 60 to about $90 \%$ for the bromine-containing species (halons and $\mathrm{CH}_{3} \mathrm{Br}$ ). The uncertainty in the lifetimes is responsible for roughly half of the total uncertainty for each species. Compared with the values defined in the Montreal Protocol, the new ODPs, based on the lifetimes from SPARC (2013) and fractional release values and $\alpha$ from WMO (2011), result in statistically significant different values for CFC-12, CFC-114, CFC-115 and $\mathrm{CCl}_{4}$, under the assumption that the ODPs in the protocol have zero uncertainty. The change in the ODP of HCFC-22 is also statistically significant, but only for the most likely uncertainty range.

Recently, Laube et al. (2013) reported new fractional release values for several ODSs (see Sect. 2.1). The use of these values also affects the semiempirical ODPs as shown in Table 3. The different fractional release values alone have the largest impact on the HCFCs with a decrease by about $30 \%$ for HCFC-22 and HCFC-141b and by about $60 \%$ for HCFC$142 \mathrm{~b}$ when compared with using WMO (2011) fractional release values. Using both the lifetimes from SPARC (2013) and the fractional release values from Laube et al. (2013) results in small changes in ODPs of most species compared with the values reported in WMO (2011). The ODPs of the HCFCs show larger changes (decreases); the ODP of HCFC-22 decreases by $37 \%$, of HCFC-141b by $40 \%$, and of HCFC-142b by $64 \%$. Compared with the ODPs in the Montreal Protocol, the fractional release values from Laube et al. (2013) and the lifetimes from SPARC (2013) result in statistically significant differences for only HCFC-22 and HCFC-142b, again under the assumption that the ODPs of the Montreal Protocol have zero uncertainty. The changes in the ODPs of $\mathrm{CCl}_{4}, \mathrm{CH}_{3} \mathrm{CCl}_{3}$, and HCFC-141b are also statistically significant, but only for the most likely uncertainty range.

ODP-weighted emissions can be calculated by multiplying emissions directly with ODPs, but this is not the best approach here for calculating uncertainties in the ODPweighted emissions due to the correlation between the ODP values themselves and the emissions. This occurs because the uncertainties in the ODS emissions estimated from atmospheric observations of concentration and in the ODPs both depend on the uncertainties in the lifetimes. These uncertainties will be anticorrelated to some degree and will thus lead to less uncertainty in the calculation of ODP-weighted emissions than if they were uncorrelated. The anticorrelation is generally smaller in the future than in the past because of the different approach to calculating emissions during these periods. The ODP-weighted emissions (Fig. 9) have therefore been calculated applying uncertainties to all parameters using the box model and Monte Carlo analysis, which allows for this anticorrelation to be properly included. The ODPweighted anthropogenic emissions peaked around 1988 at $1.3 \mathrm{Mt}$ CFC-11 eq. $\mathrm{yr}^{-1}$ with a possible range from 0.9 to $1.8 \mathrm{Mt}$ CFC-11 eq. $\mathrm{yr}^{-1}$. After this peak the anthropogenic emissions decrease strongly and past 2025 the contributions from natural $\mathrm{CH}_{3} \mathrm{Br}$ and $\mathrm{CH}_{3} \mathrm{Cl}$ emissions become larger than those from the anthropogenic emissions.

The uncertainties in ODP-weighted emissions (Fig. 9) vary from about 20 to $30 \%$ before 1990 and are dominated by the uncertainty in the ODPs of the CFCs and $\mathrm{CCl}_{4}$. With large decreases in the emissions of these ODSs in the 1990s, the uncertainty in ODP-weighted emissions increases to 40$60 \%$ by 2010 , because of a larger relative contribution from halon emissions.

\section{Global warming potentials}

For species such as ODSs and other halocarbons, for which the response of a pulse emission can be described by a single exponential decay function, the GWP can be calculated using the following equation:

$\mathrm{GWP}_{i}=\frac{R_{i}}{R_{\mathrm{CO}_{2}}} \frac{M_{\mathrm{CO}_{2}}}{M_{i}} \frac{1}{I_{\mathrm{CO}_{2}}} \tau_{i}\left(1-e^{-T_{\mathrm{H} / \tau_{i}}}\right)$ 
Table 3. ODPs and their uncertainties. The ODPs shown are those included in the Montreal Protocol, the semiempirical values reported in WMO (2011), and the semiempirical values calculated here using the lifetimes from SPARC (2013) and fractional release values from WMO (2011) and Laube et al. (2013).

\begin{tabular}{|c|c|c|c|c|c|c|c|}
\hline & \multirow[t]{2}{*}{ Montreal Protocol } & \multirow[t]{2}{*}{ WMO (2011) } & \multirow{2}{*}{$\begin{array}{c}\text { Fractional release WMO } \\
\text { Lifetimes SPARC }\end{array}$} & \multicolumn{2}{|c|}{ Fractional release Laube } & \multicolumn{2}{|c|}{ Uncertainties (95\% confidence interval) } \\
\hline & & & & Lifetimes WMO & Lifetimes SPARC & Possible $( \pm)$ & Most likely $( \pm)$ \\
\hline CFC-11 & 1 & 1 & 1 & 1 & 1 & & \\
\hline CFC-12 & 1.0 & 0.82 & 0.73 & 0.91 & 0.81 & $34 \%$ & $30 \%$ \\
\hline CFC-114 & 1.0 & 0.58 & 0.50 & & & $37 \%$ & $30 \%$ \\
\hline CFC-115 & 0.6 & 0.57 & 0.26 & & & $34 \%$ & $32 \%$ \\
\hline $\mathrm{CCl} 4$ & 1.1 & 0.82 & 0.82 & 0.83 & 0.82 & $34 \%$ & $30 \%$ \\
\hline $\mathrm{CH}_{3} \mathrm{CCl}_{3}$ & 0.1 & 0.16 & 0.14 & 0.20 & 0.17 & $52 \%$ & $36 \%$ \\
\hline HCFC-142b & 0.065 & 0.06 & 0.057 & 0.025 & 0.023 & $67 \%$ & $56 \%$ \\
\hline Halon-1211 & 3.0 & 7.9 & 6.9 & 8.9 & 7.7 & $90 \%$ & $82 \%$ \\
\hline Halon-1202 & & 2.2 & 1.7 & & & $96 \%$ & $88 \%$ \\
\hline Halon-1301 & 10.0 & 15.9 & 15.2 & 19.8 & 19.0 & $61 \%$ & $57 \%$ \\
\hline Halon-2402 & 6.0 & 13.0 & 15.7 & & & $80 \%$ & $71 \%$ \\
\hline $\mathrm{CH}_{3} \mathrm{Br}$ & 0.6 & $0.66^{\mathrm{b}}$ & 0.50 & & & $78 \%$ & $69 \%$ \\
\hline
\end{tabular}

a In the calculation of the uncertainties a correlation coefficient of 0.9 is used for the lifetime uncertainties of CFC-11 and the other $\mathrm{CFCs}_{\mathrm{C}} \mathrm{CCl}{ }_{4}$ and halon-1301.

b This value was based on a best-estimate lifetime of $\mathrm{CH}_{3} \mathrm{Br}$ of $0.8 \mathrm{yr}$.

Table 4. GWPs and their uncertainties. The GWPs shown are the values reported in WMO (2011) and the values and uncertainties calculated here using the same radiative efficiencies and absolute GWPs of $\mathrm{CO}_{2}$, but the lifetimes from SPARC (2013). The GWPs of IPCC (2013) are also given for reference.

\begin{tabular}{|c|c|c|c|c|c|c|c|c|c|c|c|}
\hline & \multicolumn{3}{|c|}{ WMO (2011) } & \multicolumn{3}{|c|}{$\begin{array}{c}\text { This work based on lifetimes } \\
\text { of SPARC (2013) }\end{array}$} & \multicolumn{3}{|c|}{$\begin{array}{c}\text { Possible (most likely) } \\
\text { uncertainty ranges }( \pm)(95 \% \text { confidence interval) }\end{array}$} & \multicolumn{2}{|c|}{$\operatorname{IPCC}(2013)^{*}$} \\
\hline & $20 \mathrm{yr}$ & $100 \mathrm{yr}$ & $500 \mathrm{yr}$ & $20 \mathrm{yr}$ & $100 \mathrm{yr}$ & $500 \mathrm{yr}$ & $20 \mathrm{yr}$ & $100 \mathrm{yr}$ & $500 \mathrm{yr}$ & $20 \mathrm{yr}$ & $100 \mathrm{yr}$ \\
\hline CFC-11 & 6730 & 4750 & 1620 & 6940 & 5260 & 1870 & $22 \%(21 \%)$ & $40 \%(31 \%)$ & $53 \%(38 \%)$ & 6900 & 4660 \\
\hline CFC-12 & 11000 & 10900 & 5200 & 11030 & 10990 & 5300 & $20 \%(20 \%)$ & $30 \%(28 \%)$ & $43 \%(34 \%)$ & 10800 & 10200 \\
\hline CFC-113 & 6540 & 6130 & 2690 & 6610 & 6390 & 2930 & $20 \%(20 \%)$ & $31 \%(28 \%)$ & $45 \%(34 \%)$ & 6490 & 5820 \\
\hline CFC-114 & 7890 & 9180 & 6330 & 7900 & 9170 & 6310 & $20 \%$ & $28 \%$ & $36 \%$ & 7710 & 8590 \\
\hline CFC-115 & 5290 & 7230 & 9120 & 5250 & 6930 & 7520 & $20 \%$ & $27 \%$ & $34 \%$ & 5860 & 7670 \\
\hline $\mathrm{CCl}_{4}$ & 2700 & 1400 & 435 & 2830 & 1590 & 502 & $22 \%(21 \%)$ & $40 \%(34 \%)$ & $46 \%(39 \%)$ & 3480 & 1730 \\
\hline $\mathrm{CH}_{3} \mathrm{CCl}_{3}$ & 506 & 146 & 45 & 488 & 141 & 43 & $21 \%$ & $28 \%$ & $32 \%$ & 578 & 160 \\
\hline HCFC-22 & 5130 & 1790 & 545 & 5170 & 1810 & 550 & $28 \%$ & $42 \%$ & $44 \%$ & 5280 & 1760 \\
\hline HCFC-141b & 2240 & 717 & 218 & 2280 & 733 & 223 & $29 \%$ & $40 \%$ & $43 \%$ & 2550 & 782 \\
\hline HCFC-142b & 5390 & 2220 & 678 & 5520 & 2320 & 709 & $24 \%$ & $38 \%$ & $41 \%$ & 5020 & 1980 \\
\hline Halon-1211 & 4750 & 1890 & 575 & 4750 & 1890 & 575 & $35 \%$ & $62 \%$ & $65 \%$ & 4590 & 1750 \\
\hline Halon-1202 & & & & & & & & & & 848 & 231 \\
\hline Halon-1301 & 8480 & 7140 & 2760 & 8610 & 7570 & 3060 & $20 \%(20 \%)$ & $31 \%(29 \%)$ & $40 \%(36 \%)$ & 7800 & 6290 \\
\hline Halon-2402 & 3680 & 1640 & 503 & 4170 & 2250 & 704 & $23 \%$ & $43 \%$ & $48 \%$ & 3440 & 1470 \\
\hline $\mathrm{CH}_{3} \mathrm{Br}$ & 19 & 5 & 2 & 17 & 5 & 1 & $39 \%$ & $43 \%$ & $46 \%$ & 9 & 2 \\
\hline $\mathrm{CH}_{3} \mathrm{Cl}$ & 45 & 13 & 4 & 41 & 12 & 4 & $41 \%$ & $45 \%$ & $47 \%$ & 45 & 12 \\
\hline
\end{tabular}

where $R_{i}$ is the radiative efficiency of species $i$ $\left(\mathrm{W} \mathrm{m}^{-2} \mathrm{ppb}^{-1}\right), I_{\mathrm{CO}_{2}}$ the integral of the $\mathrm{CO}_{2}$ response function over the time horizon, $M_{i}$ the molecular weight $\left(\mathrm{g} \mathrm{mol}^{-1}\right), T_{\mathrm{H}}$ the time horizon considered (years), and $\tau_{i}$ the lifetime (years). The parameters for $\mathrm{CO}_{2}\left(R_{\mathrm{CO}_{2}}\right.$ and $\left.I_{\mathrm{CO}_{2}}\right)$ are taken from IPCC (2007). The fractional uncertainty follows from the partial derivatives of the GWP with respect to the various variables:

$$
\begin{aligned}
& \left(\frac{\sigma_{\mathrm{GWP}_{i}}}{\mathrm{GWP}_{i}}\right)^{2} \approx\left(\frac{\sigma_{R_{i}}}{R_{i}}\right)^{2}+\left(\frac{\sigma_{R_{\mathrm{CO}_{2}}}}{R_{\mathrm{CO}_{2}}}\right)^{2}+\left(\frac{\sigma_{I_{\mathrm{CO}_{2}}}}{I_{\mathrm{CO}_{2}}}\right)^{2} \\
& +\left(\frac{\sigma_{\tau_{i}}}{\tau_{i}}\right)^{2}\left(1-\frac{T_{\mathrm{H}}}{\tau_{i}} \frac{e^{-T_{\mathrm{H} / \tau_{i}}}}{\left(1-e^{-T_{\mathrm{H} / \tau_{i}}}\right)}\right)^{2},
\end{aligned}
$$

where $\sigma$ is the standard deviation of a quantity. An uncertainty of $\pm 5 \%(1 \sigma)$ (IPCC, 2001) is used for the radiative efficiency $R_{i}$ and uncertainties of $\pm 9, \pm 13$, and $\pm 15 \%(1 \sigma)$ are used for the absolute GWP of $\mathrm{CO}_{2}\left(\sim R_{\mathrm{CO}_{2}} \cdot I_{\mathrm{CO}_{2}}\right)$ with 


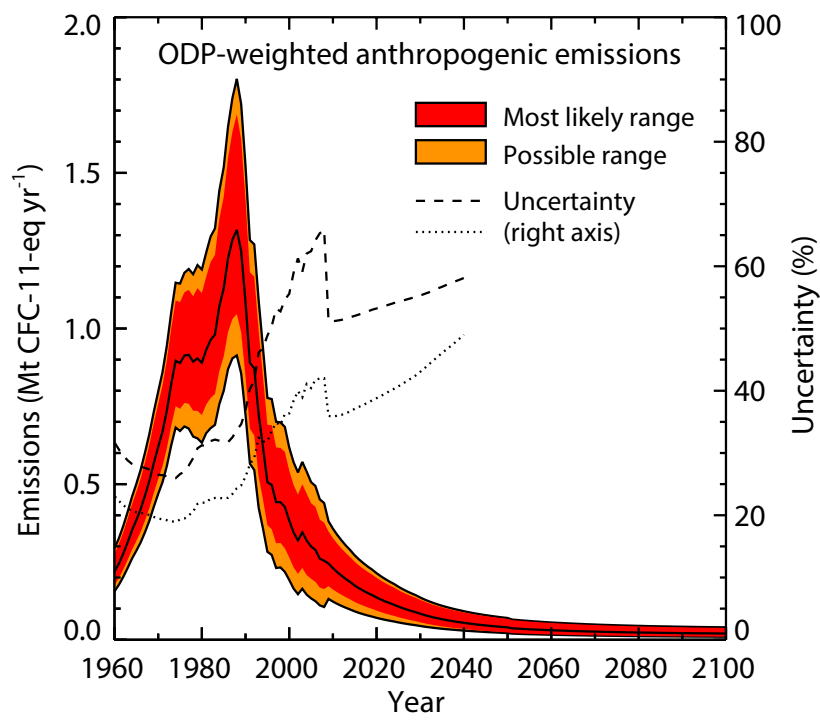

Fig. 9. ODP-weighted anthropogenic emissions from 1960 to 2100 using the lifetimes from SPARC (2013) and with uncertainties applied to all parameters. Shown are the median values and $95 \%$ confidence interval based on the possible (orange) and most likely (red) uncertainty ranges in lifetimes. The relative uncertainties $(95 \%$ confidence interval) are also shown as percentages of the median for the possible (dashed line) and most likely (dotted line) ranges until 2040, when the emissions become very small. The contribution from natural emissions of $\mathrm{CH}_{3} \mathrm{Br}$ and $\mathrm{CH}_{3} \mathrm{Cl}$ (not shown in the figure) is in total about $0.12 \mathrm{Mt} \mathrm{CFC-11} \mathrm{eq.} \mathrm{yr}^{-1}$.

time horizons of 20,100, and $500 \mathrm{yr}$, respectively (based on IPCC (2007) and Joos et al. (2013); see Table 2).

The GWPs and their uncertainties are shown in Table 4. The GWPs calculated using the lifetimes from SPARC (2013) are within $\pm 5 \%$ from those based on the lifetimes from WMO (2011) for most species. Larger differences are seen for the GWP (100 yr) of CFC-11 (+11\%), $\mathrm{CCl}_{4}$ $(+14 \%)$, and some halons and for the GWP $\left(T_{\mathrm{H}}=500 \mathrm{yr}\right)$ for several species.

The uncertainty in the GWP depends both on the lifetime and time horizon (see last term in Eq. 7). In general, the longer the lifetime of a species, the smaller the uncertainty in GWP, and the longer the time horizon the larger the uncertainty (see also Joos et al., 2013; Reisinger et al., 2010). For example, if the time horizon is much smaller than the lifetime, the amount of the emission pulse that remains in the atmosphere over the time horizon is independent of the lifetime. On the other hand, the integrated radiative effect of a pulse becomes linearly dependent on the lifetime for time horizons much longer than the lifetime. The possible uncertainty range in the GWP of CFC-11, for example, more than doubles when going from a time horizon of 20 to $500 \mathrm{yr}$.

The average GWP uncertainty (possible range; $95 \%$ confidence interval) is about $\pm 26, \pm 38$, and $\pm 44 \%$ for a 20 , 100 , and $500 \mathrm{yr}$ time horizon, respectively, but there is a large

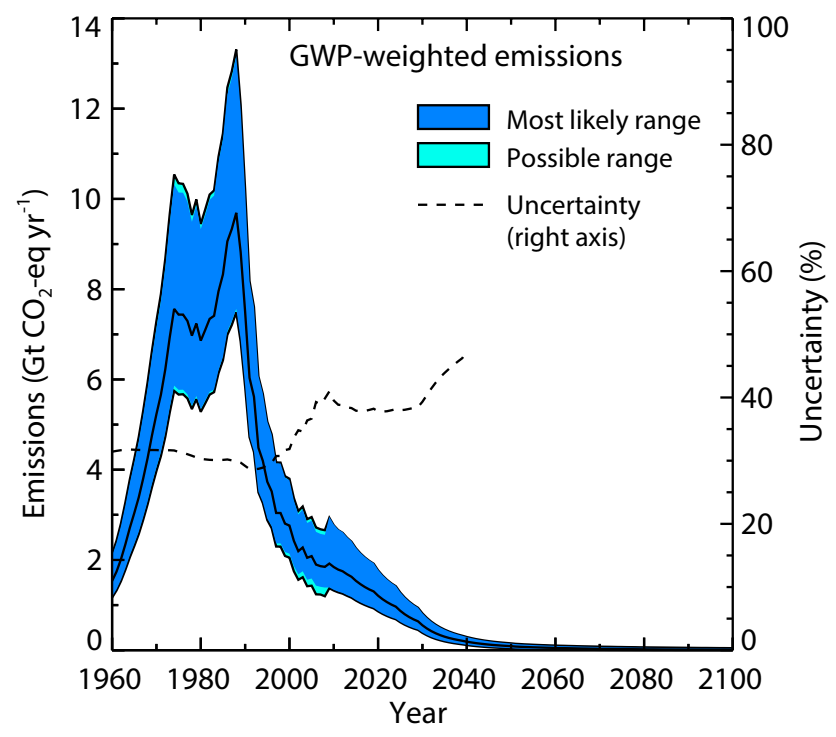

Fig. 10. GWP-weighted anthropogenic emissions from 1960 to 2100 using the lifetimes from SPARC (2013) and with uncertainties applied to all parameters. Shown are the median values and $95 \%$ confidence interval based on the possible (light blue) and most likely (dark blue) uncertainty ranges in lifetimes. The relative uncertainties (95\% confidence interval) are also shown as percentages (dashed line) until 2040, when the emissions become very small. The contribution from natural emissions of $\mathrm{CH}_{3} \mathrm{Br}$ and $\mathrm{CH}_{3} \mathrm{Cl}$ is negligible.

spread in the uncertainties among the species, depending on the uncertainty in the lifetime and the value of the lifetime, itself. The uncertainty in the lifetime is the major contributor for most species, especially for 100 and $500 \mathrm{yr}$ time horizons, followed by the uncertainty in the absolute GWP of $\mathrm{CO}_{2}$.

IPCC (2007) quotes an uncertainty in GWPs of $\pm 35 \%$ for a $90 \%$ confidence interval, which is probably based on a statement in IPCC (1995) that "suggests uncertainties of less than $\pm 35 \%$ " based on a simple calculation. The uncertainty of $\pm 35 \%$ agrees with the average uncertainty of $\pm 38 \%$ derived here for a time horizon of $100 \mathrm{yr}$. The uncertainty in IPCC (2007) does not agree with the average uncertainty of \pm 26 and $\pm 44 \%$ for GWPs for a time horizon of 20 and $500 \mathrm{yr}$, respectively, and it does also not take into account the large range of uncertainties (for example, 27$62 \%$ for $\left.\operatorname{GWP}\left(T_{\mathrm{H}}=100 \mathrm{yr}\right)\right)$ for different species. In IPCC (2013) an uncertainty of 20 and $35 \%$ is given for CFC-11 for a time horizon of 20 and $100 \mathrm{yr}$, respectively, and of 20 and $30 \%$ for CFC-12. These uncertainties agree reasonably well with our calculated values. The GWPs presented in Table 4 are derived for the current atmosphere composition although changes in the composition will certainty affect GWPs. For example, increases in the $\mathrm{CO}_{2}$ mixing ratio for $5 \mathrm{yr}(\sim 10 \mathrm{ppm})$ will increase the GWPs of Table 4 by about $2.5 \%$. 
The GWP-weighted emissions have been calculated in the same way as the ODP-weighted emissions using the box model and Monte Carlo analysis. The GWP-weighted emissions (Fig. 10) peaked around 1988 at $9.7 \mathrm{Gt} \mathrm{CO}_{2}$ eq. $\mathrm{yr}^{-1}$ with a possible 7.5 to $13.3 \mathrm{Gt}^{\mathrm{CO}_{2}}$ eq. $\mathrm{yr}^{-1}$ range. These emissions dropped to $1.7 \mathrm{Gt} \mathrm{CO}_{2}$ eq. $\mathrm{yr}^{-1}(1.2-2.5)$ by 2013 and are projected to continue to decrease. The uncertainty in the GWP-weighted emissions is about $30 \%$ up to 2000 as it is dominated by the contribution from CFC-12. After 2000 the uncertainty increases to about $40 \%$ since the emissions of CFC-12 decrease and the uncertainty is dominated by contributions from HCFC22.

\section{Discussion}

A new ODS scenario including uncertainties has been derived. These uncertainties are primarily controlled by the lifetime uncertainties. For most parameters, the magnitude of the uncertainty does not substantially affect the overall uncertainty analysis. For example, the uncertainties in the production data, bank sizes in 2008, emission factors, observed mixing ratios, and surface factor ( $\left.F_{\text {surf }}\right)$ are not very important for uncertainties in future mixing ratios and EESC levels. For some species, however, the uncertainty in the size of the bank in 2008 is relevant for mixing ratio projections. The uncertainty in the future mixing ratios of halon- 1301 is determined roughly equally by the uncertainty in the lifetime and in the size of the bank of 2008, which is a result of the relatively small uncertainty in the lifetime of $9 \%$ compared with an uncertainty in the size of the bank of $20 \%$. For HCFC-141b and HCFC-142b the uncertainty in the size of bank in 2008 makes up about $20 \%$ of the total uncertainty in the mixing ratios in 2050. To understand the effects of the uncertainty in the size of the bank on future mixing ratios it is important to realize how the size of the bank in 2008 is used in the model. As explained in Sect. 2.1 this bank size is not only a key starting point for the calculation of future emissions and mixing ratios, it also determines, together with the historic production data and emissions derived from observed mixing ratios, the emission factor for future emissions. Therefore, an increase of the size of the bank in 2008 results in increased future emissions and mixing ratios, but these increases as a result of the larger size of the bank are somewhat reduced by a decrease in the emission factor, which results is lower emissions. For HCFCs future emissions not only originate from the bank in 2008, but also from future production. Future cumulative HCFC production in the baseline scenario from WMO (2011) is 2-5 times larger for the different HCFCs than the size of the bank in 2008. The future emissions are also affected by the change in emission factor as a result of the uncertainty in the size of the bank. The overall effect of an increase in the size of the bank in 2008 is a decrease in emissions for $10-20 \mathrm{yr}$, followed by an increase afterwards. The specific turnover point is determined by the ratio of the future production and the size of the bank as well as by the emission fraction and thus by the types of applications in which the ODS is used.

The magnitude of the uncertainty of the size of bank of CFCs hardly affects the total uncertainty in future mixing ratios, since for these species the size of the bank in 2008 is relatively small compared with the total atmospheric burden of the ODSs and production of CFCs has almost completely ceased globally. Of the CFCs, CFC-11 has the largest bank of about $1420 \mathrm{kt}$ in 2008, but this is still a factor of four smaller than its total atmospheric burden in that year. The atmospheric burden of CFC-12 is about 30 times larger than the size of the bank in 2008.

The uncertainty in the size of the banks of CFC-115 and halon-2402 are also relevant for the total uncertainty in their future mixing ratios (contributing 20 and $10 \%$, respectively), but these ODSs contribute little to total EESC.

Future production is a quantity that is also relevant for the future mixing ratios of some ODSs. The production of the CFCs, halons, and $\mathrm{CH}_{3} \mathrm{CCl}_{3}$ has almost ceased globally, but significant production continues for HCFCs and to a lesser extent $\mathrm{CH}_{3} \mathrm{Br}$. The future ODS production in this study is taken from the baseline scenario of WMO (2011) and is assumed to be characterized by a $\pm 5 \%$ uncertainty. To put the uncertainty in future mixing ratios of the HCFCs in perspective, it can be compared with a scenario with an elimination of production of HCFCs from 2014 onward. In such a scenario the mixing ratios of HCFC-22, HCFC-141b, and HCFC- $142 \mathrm{~b}$ are reduced by 45,10 , and $8 \mathrm{ppt}$, respectively, in 2050, compared to the baseline scenario. These decreases in mixing ratios are comparable to the differences between the lower end of the uncertainty range and the baseline scenario of 35, 10, and $6 \mathrm{ppt}$, for HCFC-22, HCFC-141b, and HCFC$142 \mathrm{~b}$, respectively, in 2050, applying uncertainties to all parameters. This shows that the effect of the future production of HCFCs results in a change in mixing ratios in 2050 that is comparable to the uncertainty in mixing ratios resulting from the Monte Carlo uncertainty analysis. The effects of the future production of HCFCs on EESC levels is limited, increasing the year EESC returns to pre-1980 levels by 1$2 \mathrm{yr}$ for midlatitude conditions compared with a scenario of no future HCFC production.

Changes in the mixing ratios of other species - methane, nitrous oxide, and very short-lived species (VSLS) - and changes in temperature and atmospheric circulation due to climate change will also affect future ozone levels. This can be directly or through changes in the lifetimes of the ODSs. Changes in circulation may affect the recovery of the ozone layer by changing the age of air in the stratosphere as well as through other effects. Overall, climate change effects are currently expected to accelerate the recovery of the ozone layer at midlatitudes by a decade or more, but delay it in the Antarctic (WMO, 2011). These aspects have not been considered here, since the focus is on changes and 
uncertainties in long-lived chlorine- and bromine-containing species that are controlled by the Montreal Protocol.

\section{Conclusions}

We have shown, through a comprehensive uncertainty analysis, that the uncertainty in the lifetimes of the ODSs is the dominant term in the overall uncertainty in total EESC levels. Projections of ozone-layer recovery from depletion by ODSs depends strongly on knowledge of these lifetime estimates and uncertainties. The year EESC returns to pre-1980 levels is 2048 for midlatitude conditions and 2075 for Antarctic conditions, based on our baseline for ODS emissions and lifetimes from the SPARC (2013). Taking all uncertainties in the model parameters into account yields a range for the year EESC returns to pre-1980 levels from 2039 to 2064 ( $95 \%$ confidence interval) for midlatitude conditions and from 2061 to 2105 for Antarctic conditions. Since the current atmospheric burden of CFCs is much larger than the amounts present in existing equipment or still being produced, future mixing ratios and EESC levels primarily depend on the lifetimes and their uncertainties. The lower end of the uncertainty range in EESC is comparable with the EESC levels resulting from a hypothetical scenario with a cease in anthropogenic ODS emissions in 2014. The upper end of the range corresponds with an extra emission about $7 \mathrm{Mt}$ CFC-11 eq. in 2015. This is equivalent to about 2 times the cumulative anthropogenic emissions of all ODSs, or about 12 times the cumulative HCFC emissions from 2014 to 2050.

Semiempirical ODPs calculated using the lifetimes from SPARC (2013) and fractional release values from WMO (2011) (mostly based on Newman et al. (2006)) are for most species up to $25 \%$ lower than the data reported in WMO (2011) using the lifetimes from that report, mainly as a result of the estimate of the CFC-11 lifetime increasing by $16 \%$. The ODP of halon-2402 increases by $20 \%$ and of CFC- 115 decreases by $50 \%$. The uncertainties (95\% confidence interval) in ODPs are about $30-35 \%$ for the CFCs, $55-70 \%$ for the HCFCs, and $60-90 \%$ for the brominecontaining species (halons and $\mathrm{CH}_{3} \mathrm{Br}$ ). Compared with the values defined in the Montreal Protocol, the lifetimes from SPARC (2013) and fractional release values and $\alpha$ from WMO (2011) result in statistically significant differences in the ODPs of CFC-12, CFC-114, CFC-115 and $\mathrm{CCl}_{4}$, under the assumption that the ODPs in the Montreal Protocol have zero uncertainty.

Based on our analysis, we have evaluated the radiative forcing of the ODSs. Their radiative forcing is $0.32 \mathrm{~W} \mathrm{~m}^{-2}$ (ranges from 0.30 to $0.34,95 \%$ confidence interval) in 2010 and is expected to decrease to $0.20 \mathrm{~W} \mathrm{~m}^{-2}(0.17-0.23)$ in 2050 and to $0.10 \mathrm{~W} \mathrm{~m}^{-2}(0.07-0.14)$ in 2100 . The GWPs calculated using the lifetimes from SPARC (2013) are within $\pm 5 \%$ from those based on the lifetimes from WMO (2011) for most species. Larger differences are calculated for the
GWPs (100 yr) of CFC-11 (+11\%) and $\mathrm{CCl}_{4}(+14 \%)$. The average uncertainty for the GWPs of the ODSs is about \pm 26 , \pm 38 , and $\pm 44 \%$ for a 20,100 , and $500 \mathrm{yr}$ time horizon, respectively.

Two files are included in the Supplement with the results of a full uncertainty analysis calculation of production, banks, emissions, EESC, radiative forcing, ODP- and GWPweighted emissions from 1950 to 2120 .

\section{Supplementary material related to this article is available online at http://www.atmos-chem-phys.net/14/ 2757/2014/acp-14-2757-2014-supplement.zip.}

Acknowledgements. We thank the steering committee of the WCRP/SPARC lifetimes report, Malcolm Ko (NASA/LARC), Stefan Reimann (EMPA), and Paul Newman and Susan Strahan (NASA/GSFC) for providing the new lifetimes data at an early stage, Qing Liang (NASA/GSFC) for providing modeled trends in lifetimes, and Steve Montzka (NOAA/ESRL) for a useful discussion.

Edited by: W. Thomas

\section{References}

Brown, A. T., Volk, C. M., Schoeberl, M. R., Boone, C. D., and Bernath, P. F.: Stratospheric lifetimes of CFC-12, $\mathrm{CCl}_{4}, \mathrm{CH} 4$, $\mathrm{CH}_{3} \mathrm{Cl}$ and $\mathrm{N}_{2} \mathrm{O}$ from measurements made by the Atmospheric Chemistry Experiment-Fourier Transform Spectrometer (ACEFTS), Atmos. Chem. Phys., 13, 6921-6950, doi:10.5194/acp6913-6921-2013, 2013.

Daniel, J. S. and Velders, G. J. M. (Coordinating lead authors), Douglas, A. R., Forster, P. M. D., Hauglustaine, D. A., Isaksen, I. S. A., Kuijpers, L. J. M., McCulloch, A., and Wallington, T. J.: Halocarbon scenarios, ozone depletion potentials, and global warming potentials, Chapter 8 of Scientific Assessment of Ozone Depletion: 2006, Global Ozone Research and Monitoring Project - Report No. 50, World Meteorological Organization, Geneva, Switzerland, 2007.

Daniel, J. S. and Velders, G. J. M. (Coordinating lead authors), Morgenstern, O., Toohey, D. W., Wallington, T. J., and Wuebbles, D. J.: A focus on information and options for policymakers, Chapter 5 of Scientific Assessment of Ozone Depletion: 2010, Global Ozone Research and Monitoring Project - Report No. 52, World Meteorological Organization, Geneva, Switzerland, 2011.

Daniel, J. S., Solomon, S., and Albritton, D. L.: On the evaluation of halocarbon radiative forcing and global warming potentials, J. Geophys. Res., 100, 1271-1285, 1995.

Daniel, J. S., Velders, G. J. M., Solomon, S., McFarland, M., and Montzka, S. A.: Present and future sources and emissions of halocarbons: Towards new constraints, J. Geophys. Res., 112, D02301, doi:10.1029/2006JD007275, 2007.

Daniel, J. S., Flemming, E. L., Portmann, R. W., Velders, G. J. M., Jackman, C. H., and Ravishankara, A. R.: Options to accelerate ozone recovery: ozone and climate benefits, Atmos. Chem. Phys., 10, 7697-7707, doi:10.5194/acp-7697-2010, 2010. 
Douglas, A. R., Stolarski, R. S., Schoeberl, M. R., Jackman, C. H., Gupta, M. L., Newman, P. A., Nielsen, J. E., and Flemming, E. L.: Relationship of loss, mean age of air and the distribution of CFCs to stratospheric circulation and implications for atmospheric lifetimes, J. Geophys. Res., 113, D14309, doi:10.1029/2007JD009575, 2008.

Fisher, D. A., Hales, C. H., Filkin, D. L., Ko, M. K. W., Sze, N. D., Connell, P. S., Wuebbles, D. J., Isaksen, I. S. A., and Stordal, F.: Model calculations of the relative effects of CFCs and their replacements on stratospheric ozone, Nature, 344, 508-512, 1990.

Hall, T. M.: Path histories and timescales in stratospheric transport: Analysis of an idealized model, J. Geophys. Res., 105, 2281122823, 2000.

IPCC: Climate Change 1994: Radiative Forcing of Climate Change, Report of the Intergovernmental Panel on Climate Change, edited by: Houghton, J. T., Filho, L. G. M., Bruce, J., Lee, H., Callander, B., Haiter, E., Harris, N., and Maskell, K., Cambridge University Press, Cambridge, UK, and New York, NY, USA, 339 pp., 1995.

IPCC: Climate change 2001: The scientific basis, edited by: Houghton, J. T., Ding, Y., Griggs, D. J., Noguer, M., Linden, P. J. v. d., Dai, X., Maskell, K., and Johnson, C. A., Cambridge Univ Press, Cambridge, UK and New York, 994 pp., 2001.

IPCC: Climate Change 2007: The physical science basis, edited by: Solomon, S., Qin, D., Manning, M., Chen, Z., Marquis, M., Averyt, K. B., Tignor, M., and Miller, H. L., Cambridge Univ Press, Cambridge, UK and New York, 996 pp., 2007.

IPCC: Climate Change 2013: The physical science basis, edited by: Stocker, T. F., and Qin, D., Cambridge Univ Press, Cambridge, UK and New York, 1535 pp., 2013.

IPCC/TEAP: Special report: Safeguarding the ozone layer and the global climate system: Issues related to hydrofluorocarbons and perfluorocarbons, edited by: Metz, B., Kuijpers, L., Solomon, S., Andersen, S. O., Davidson, O., Pons, J., de Jager, D., T., K., Manning, M., and Meyer, L., Cambridge Univ Press, New York, 478 pp., 2005.

Joos, F., Roth, R., Fuglestvedt, J. S., Peters, G. P., G., E. I., von Bloh, W., Brovkin, V., Burke, E. J., Eby, M., Edwards, N. R., Friedrich, T., Frolicher, T. L., Halloran, P. R., Holden, P. B., C., J., Kleinen, T., Mackenzie, F. T., Matsumoto, K., Meinshausen, M., Plattner, G.-K., Reisinger, A., Segschneider, J., Shaffer, G., Steinacher, M., Strassmann, K., Tanaka, K., Timmermann, A., and Weaver, A. J.: Carbon dioxide and climate impulse response functions for the computation of greenhouse gas metrics: a multi-model analysis, Atmos. Chem. Phys., 13, 2793-2825, doi:10.5194/acp13-2793-2013, 2013.

Laube, J. C., Keil, A., Bonisch, H., Engel, A., Rockmann, T., Volk, C. M., and Sturges, W. T.: Observation-based assessment of stratospheric fractional release, lifetimes, and ozone depletion potentials of ten important source gases, Atmos. Chem. Phys., 13, 2779-2791, doi:10.5194/acp-13-2779-2013, 2013.

Martinerie, P., Nourtier-Mazauric, E., Barnola, J.-M., Sturges, W. T., Worton, D. R., Atlas, E., Gohar, L. K., Shine, K. P., and Brasseur, G. P.: Long-lived halocarbon trends and budgets from atmospheric chemistry modelling constrained with measurements in polar firn, Atmos. Chem. Phys., 9, 3911-3934, doi:10.5194/acp-13-2779-2013, 2009.

McKay, M. D., Beckman, R. J., and Conover, W. J.: A comparison of three methods for selecting values of input variables in the analysis of output from a computer code, Technometrics, 21, 239-245, doi:10.2307/1268522, 1979.

Meinshausen, M., Smith, S. J., Calvin, K., Daniel, J. S., Kainuma, M. L. T., Lamarque, J.-F., Matsumoto, K., Montzka, S. A., Raper, S. C. B., Riahi, K., Thomson, A., Velders, G. J. M., and van Vuuren, D. P.: The RCP greenhouse gas concentrations and their extensions from 1765 to 2300, Climatic Change, 109, 213-241, doi:10.1007/s10584-011-0156-z, 2011.

Minschwaner, K., Hoffmann, L., Brown, A., Riese, M., Muller, R., and Bernath, P. F.: Stratospheric loss and atmospheric lifetimes of CFC-11 and CFC-12 derived from satellite observations, Atmos. Chem. Phys., 13, 4253-4263, doi:10.5194/acp-13-42532013, 2013.

Montzka, S. A. and Reimann, S. (Coordinating lead authors), Engel, A., Kruger, K., O’Doherty, S., and Sturges, W. T.: Ozonedepleting substances (ODSs) and related chemicals, Chapter 1 of Scientific Assessment of Ozone Depletion: 2010, Global Ozone Research and Monitoring Project - Report No. 52, World Meteorological Organization, Geneva, Switzerland, 2011.

Montzka, S. A., Butler, J. H., Elkins, J. W., Thompson, T. M., Clarke, A. D., and Lock, L. T.: Present and future trends in the atmospheric burden of ozone-depleting halogens, Nature, 398, 690-694, 1999.

Montzka, S. A., Dlugokencky, E. J., and Butler, J. H.: Non$\mathrm{CO}_{2}$ greenhouse gases and climate change, Nature, 476, 43-50, doi:10.1038/nature10322, 2011.

Newman, P. A., Nash, E. R., Kawa, S. R., Montzka, S. A., and Schauffler, S. M.: When will the Antarctic ozone hole recover?, Geophys. Res. Lett., 33, L12814, doi:12810.11029/12005GL025232, 2006.

Newman, P. A., Daniel, J. S., Waugh, D. W., and Nash, E. R.: A new formulation of equivalent effective stratospheric chlorine (EESC), Atmos. Chem. Phys., 7, 4537-4552, doi:10.5194/acp7-4537-2007, 2007.

Prinn, R. G., Weiss, R. F., Fraser, P. J., Simmonds, P. G., Cunnold, D. M., Alyea, F. N., O'Doherty, S., Salameh, P., Miller, B. R., Huang, J., Wang, R. H. J., Hartley, D. E., Harth, C., Steele, L. P., Sturrrock, G., Midgley, P. M., and McCulloch, A.: A history of chemically and radiatively important gases in air deduced from ALE/GAGE/AGAGE, J. Geophys. Res., 105, 17751717792, 2000.

Ramanathan, V.: Greenhouse effect due to chlorofluorocarbons: Climate implications, Science, 190, 50-51, 1975.

Reisinger, A., Meinshausen, M., Manning, M., and Bodeker, G. E.: Uncertainties of global warming metrics: $\mathrm{CO}_{2}$ and $\mathrm{CH}_{4}$, Geophys. Res. Lett., 37, L14707, doi:10.1029/2010GL043803, 2010.

Rigby, M., Prinn, R., O’Doherty, S., Montzka, S. A., McCulloch, A., Harth, C. M., Muhle, J., Salameh, P. K., Weiss, R. F., Young, D., Simmonds, P. G., Hall, B. D., Dutton, G. S., Nance, D., Mondeel, D. J., Elkins, J. W., Krummel, P. B., Steele, L. P., and Fraser, P. J.: Re-evaluation of the lifetimes of the major CFCs and $\mathrm{CH} 3 \mathrm{CCl} 3$ using atmospheric trends, Atmos. Chem. Phys., 13, 2691-2702, doi:10.5194/acp-13-2691-2013, 2013.

Solomon, S. and Albritton, D. L.: Time-dependent ozone depletion potentials for short- and long-term forecasts, Nature, 357, 33-37, 1992.

Solomon, S., Mills, M., Heidt, L. E., Pollock, W. H., and Tuck, A. F.: On the evaluation of ozone depletion potentials, J. Geophys. Res., 97, 825-842, 1992. 
SPARC: Report on the lifetimes of stratospheric ozone-depleting substances, their replacements, and related species, edited by: Ko, M., Newman, P., Reimann, S., Strahan, S., SPARC Report No. 6, WCRP-15, Zurich, Switzerland, 2013.

UNEP: Task force decision XX/8 report, Assessment of alternatives to HCFCs and HFCs and update of the TEAP 2005 supplement report data, United Nations Environment Programme, Nairobi, Kenya, 2009.

UNEP: Production and consumption of ozone depleting substances under the Montreal Protocol, available at: http://www.unep.org/ ozone (last access: February 2010), United Nations Environment Programme, Nairobi, Kenya, 2010.

UNEP: 2010 Report of the refrigeration, air conditioning and heat pumps Technical Options Committee of the Technology and Economic Assessment Panel (TEAP), United Nations Environment Programme, Nairobi, Kenya, 2011.

Velders, G. J. M., Andersen, S. O., Daniel, J. S., Fahey, D. W., and McFarland, M.: The importance of the Montreal Protocol in protecting climate, Proc. Nat. Acad. Sci., 104, 4814-4819, doi:10.1073/pnas.0610328104, 2007.

WMO: Scientific Assessment of Ozone Depletion: 1994, Global Ozone Research and Monitoring Project - Report No. 37, World Meteorological Organization (WMO), Geneva, Switzerland, 1995.
WMO: Scientific Assessment of Ozone Depletion: 1998, Global Ozone Research and Monitoring Project - Report No. 44, World Meteorological Organization (WMO), Geneva, Switzerland, 1999.

WMO: Scientific Assessment of Ozone Depletion: 2002, Global Ozone Research and Monitoring Project - Report No. 47, World Meteorological Organization (WMO), Geneva, Switzerland, 2003.

WMO: Scientific Assessment of Ozone Depletion: 2006, Global Ozone Research and Monitoring Project - Report No. 50, World Meteorological Organization, Geneva, Switzerland, 2007.

WMO: Scientific Assessment of Ozone Depletion: 2010, Global Ozone Research and Monitoring Project - Report No. 52, World Meteorological Organization, Geneva, Switzerland, 2011.

Wuebbles, D. J.: Chlorocarbon emission scenarios: Potential impact on stratospheric ozone, J. Geophys. Res., 88, 1433-1443, 1983.

Yvon-Lewis, S. A. and Butler, J. H.: Effect of oceanic uptake on atmospheric lifetimes of selected trace gases, J. Geophys. Res., 107, 4414, doi:10.1029/2001JD001267, 2002.

Yvon-Lewis, S. A., Saltzman, E. S., and Montzka, S. A.: Recent trends in atmospheric methyl bromide: analysis of postMontreal Protocol variability, Atmos. Chem. Phys., 9, 59635974, doi:10.5194/acp-9-5963-2009, 2009. 